\title{
Predicting the effect of reference population on the accuracy of within, across, and multibreed genomic prediction
}

\author{
I. van den Berg, ${ }^{1,2 *}$ T. H. E. Meuwissen, ${ }^{3}$ I. M. MacLeod, ${ }^{2}$ and M. E. Goddard ${ }^{1,2}$ \\ ${ }^{1}$ Faculty of Veterinary \& Agricultural Science, University of Melbourne, 3010 Parkville, Victoria, Australia \\ ${ }^{2}$ Agriculture Victoria, AgriBio, Centre for AgriBioscience, 3083 Bundoora, Victoria, Australia \\ ${ }^{3}$ Department of Animal and Aquacultural Sciences, Norwegian University of Life Sciences, 1432 Ås, Norway
}

\section{ABSTRACT}

Genomic prediction is widely used to select candidates for breeding. Size and composition of the reference population are important factors influencing prediction accuracy. In Holstein dairy cattle, large reference populations are used, but this is difficult to achieve in numerically small breeds and for traits that are not routinely recorded. The prediction accuracy is usually estimated using cross-validation, requiring the full data set. It would be useful to have a method to predict the benefit of multibreed reference populations that does not require the availability of the full data set. Our objective was to study the effect of the size and breed composition of the reference population on the accuracy of genomic prediction using genomic BLUP and Bayes R. We also examined the effect of trait heritability and validation breed on prediction accuracy. Using these empirical results, we investigated the use of a formula to predict the effect of the size and composition of the reference population on the accuracy of genomic prediction. Phenotypes were simulated in a data set containing real genotypes of imputed sequence variants for 22,752 dairy bulls and cows, including Holstein, Jersey, Red Holstein, and Australian Red cattle. Different reference populations were constructed, varying in size and composition, to study within-breed, multibreed, and across-breed prediction. Phenotypes were simulated varying in heritability, number of chromosomes, and number of quantitative trait loci. Genomic prediction was carried out using genomic BLUP and Bayes R. We used either the genomic relationship matrix (GRM) to estimate the number of independent chromosomal segments and subsequently to predict accuracy, or the accuracies obtained from single-breed reference populations to predict the accuracies of larger or multibreed reference populations. Using the GRM overestimated

Received June 18, 2018.

Accepted December 8, 2018.

*Corresponding author: irene.vandenberg@ecodev.vic.gov.au the accuracy; this overestimation was likely due to close relationships among some of the reference animals. Consequently, the GRM could not be used to predict the accuracy of genomic prediction reliably. However, a method using the prediction accuracies obtained by cross-validation using a small, single-breed reference population predicted the accuracy using a multibreed reference population well and slightly overestimated the accuracy for a larger reference population of the same breed, but gave a reasonably close estimate of the accuracy for a multibreed reference population. This method could be useful for making decisions regarding the size and composition of the reference population.

Key words: genomic prediction, prediction accuracy, reference population, multibreed prediction, acrossbreed prediction

\section{INTRODUCTION}

Genomic prediction (Meuwissen et al., 2001) is widely used to select candidates for breeding. The accuracy of prediction is commonly estimated using cross-validation, dividing the data set in a reference and validation set, with marker effects estimated in the reference set used to predict genomic estimated breeding values (GEBV) for the validation set. The accuracy is then measured as the correlation between GEBV and phenotypes. With this approach, the accuracy can only be obtained when the full data set is available. It would be desirable to estimate the prediction accuracy before the data set is available; for example, to aid making decisions regarding the size and composition of the reference population.

Factors influencing prediction accuracy include the size of the reference population (Daetwyler et al., 2008; Goddard, 2009; Habier et al., 2010), heritability of the trait (Goddard, 2009; Daetwyler et al., 2010), relatedness between reference and validation population (de Roos et al., 2009; Wientjes et al., 2013), effective population size (Goddard, 2009), linkage disequilibrium (LD) between prediction markers and QTL (Habier et 
al., 2007; Wientjes et al., 2013), marker density (Meuwissen and Goddard, 2010; Clark et al., 2011), number of QTL (Daetwyler et al., 2010; Clark et al., 2011), and minor allele frequencies of causative mutations and prediction markers (Druet et al., 2014; Wientjes et al., 2015a). The effect of these factors on the prediction accuracy is influenced by the choice of prediction model. For example, the number of QTL influences the accuracy of Bayesian variable selection models, but not the accuracy of genomic BLUP (GBLUP; Daetwyler et al., 2010; Clark et al., 2011).

Several studies have developed formulae to estimate the accuracy as a function of the size of the reference population, heritability, number of independent chromosomal segments $\left(\boldsymbol{M}_{\boldsymbol{e}}\right.$; Goddard, 2009), and various weighting factors (Daetwyler et al., 2008, 2010; Goddard, 2009; Goddard et al., 2011; Erbe et al., 2013; Wientjes et al., 2015b). A difficulty in applying the deterministic equations is deriving the appropriate $M_{e}$. A range of formulae have been published for a priori estimates of $M_{e}$ (Goddard, 2009; Hayes et al., 2009b; Goddard et al., 2011; Lee et al., 2017), mostly relying on an estimate of the effective population size (Ne), because Ne controls the LD pattern within breed. However, although we know that the current Ne of Holstein cattle is $\sim 100$ (Young and Seykora, 1996), the LD pattern in their genomes does not match that of a constant $\mathrm{Ne}=100$ because the relatively recent ancestral population has been much larger than 100. Furthermore, we cannot predict $\mathrm{Ne}$ for a multibreed reference population without resorting to empirical estimates of LD, requiring genotypes of the animals. Other methods have been proposed to estimate $M_{e}$ from genomic relationships (Goddard et al., 2011; Lee et al., 2017), eigenvalue decomposition of the genomic relationship matrix (Misztal, 2016; Pocrnic et al., 2016a,b), or cross-validation accuracies (Erbe et al., 2013), which also requires the animals to be genotyped. Brard and Ricard (2015) compared 4 previously published formulae (Daetwyler et al., 2008; Goddard, 2009; Goddard et al., 2011; Meuwissen et al., 2013) on data from 13 publications and showed that the formulae gave different estimates of the accuracy depending on the parameters used, but none of the formulae consistently outperformed the others.

In Holstein cattle, large reference populations are used, but this is difficult to achieve in numerically small breeds and for traits that are not routinely recorded. Therefore, a method to predict the benefit of multibreed reference populations would be useful and does not currently exist. Wientjes et al. (2015b,c) adapted the formulae from Daetwyler et al. (2010) and Goddard et al. (2011) for across-population and multipopulation prediction, but this resulted in an overestimation of the accuracy, especially across populations.
Our objective was to develop a means of predicting the effect of the size and breed composition of the reference population on the accuracy. Using simulated phenotypes based on real data genotypes, we examined the effect of the breeds, number of animals, and heritability on prediction accuracy. We investigated the use of a formula to predict the effect of the size and composition of the reference population on the accuracy. Subsequently, we used published heritabilities and within-breed accuracies to predict the accuracy of a multibreed reference population for a real data set.

\section{MATERIALS AND METHODS}

\section{Simulation}

Phenotypes were simulated in a data set containing realized imputed sequence variants for 7,510 Holstein bulls, 8,619 Holstein cows, 875 Jersey bulls, 3,968 Jersey cows, 870 Red Holstein bulls, 114 Australian Red bulls, and 796 Australian Red cows. The data set was divided into reference and validation populations. For Holstein and Jersey, the oldest animals were allocated to the reference populations and the youngest animals to the validation population, with 582 and 551 Holstein and Jersey validation individuals, respectively. All Red Holstein and Australian Red bulls were used as validation populations, whereas all Australian Red cows were used as reference population. For the Holstein, Jersey, and Australian Red breeds, 2 reference populations were constructed to contain either a random subset of approximately half the number of individuals in a breed or all individuals of a breed (excluding individuals assigned to the validation population). Consequently, the within-breed reference populations contained 7,989 Holstein $(\mathbf{H}), 15,547$ Holstein $(\mathbf{H H})$, 2,323 Jersey (J), 4,310 Jersey (JJ), 384 Australian Red (R), and 769 Australian Red (RR). Multibreed reference populations were constructed by combining 7,989 Holstein and 2,323 Jersey $(\mathbf{H}+\mathbf{J})$ or 7,989 Holstein, 2,323 Jersey, and 769 Australian Red $(\mathbf{H}+\mathbf{J}+\mathbf{R R})$ individuals. An overview of the different reference and validation populations can be found in Table 1, and the population structure is shown in Figure 1 by plotting the first 2 principal components of the genomic relationship matrix. The principal component analysis used to construct this figure was done by randomly selecting 500 individuals of each breed (Holstein, Jersey, Australian Red, and Red Holstein) and subsequently using the prcomp() command in R Core Team (2015).

Genotypes contained 994,019 sequence variants in gene coding regions or $5,000 \mathrm{bp}$ up- and downstream of genes. These variants included 45,026 nonsynonymous coding variants, 578,734 variants upstream or down- 
stream of genes or in $3^{\prime}$ of $5^{\prime}$ untranslated genic regions, and 370,259 variants that were present on the $800 \mathrm{~K}$ Illumina BovineHD BeadChip (Illumina Inc., San Diego, $\mathrm{CA}$ ). More details of the genomic data set can be found in MacLeod et al. (2016).

A range of scenarios was simulated, varying the heritability $\left(\mathbf{h}^{2}\right)$, number of QTL, distribution of QTL effects, and the size of the genome (by altering the number of chromosomes used). Table 2 summarizes all scenarios. The QTL were simulated by randomly sampling $100,2,000,4,000$, or 10,000 variants from all genotyped variants. Subsequently, additive QTL effects $\left(a_{i}\right)$ were sampled from 3 normal distributions: $S=N\left(0,0.0001 \sigma_{g}^{2}\right)$, $M=N\left(0,0.001 \sigma_{g}^{2}\right), \quad$ and $L=N\left(0,0.01 \sigma_{g}^{2}\right)$, where $\sigma_{g}^{2}$ equals the additive genetic variance, $S=$ small, $M=$ medium, and $L=$ large. In the scenarios with 100 QTL, all QTL effects were drawn from $L$, and in the scenarios with 10,000 QTL all effects were drawn from $S$. In the scenarios with 2,000 QTL, a mixture distribution was simulated with 1,360, 615, and 25 QTL effects drawn from $S, M$, and $L$, respectively, whereas in the scenarios with 4,000 QTL, 3,485, 500, and 15 QTL effects were drawn from $S, M$, and $L$, respectively. All scenarios were replicated 5 times; QTL were selected out of all variants in the data set, regardless of their allele frequency and in which breeds they were segregating. Although the majority of QTL were segregating in all breeds, this was not the case for all QTL, and QTL could have different allele frequencies in different breeds. Appendix Table A1 and Figure A1 show the average number of QTL segregating in each breed for the scenario with 4,000 QTL and the distribution of allele frequencies in each breed, respectively.

The true breeding value (TBV) of individual $j$ was computed as $T B V_{j}=\sum_{i=1}^{n Q T L} x_{i j} a_{i}$, where $x_{i j}$ is the genotype of individual $j$ coded as 0,1 , and 2 for QTL $i$; $a_{i}$ the additive effect of QTL $i$; and $n Q T L$ is the number of
QTL. Subsequently, an environmental effect was sampled across populations from a normal distribution and added to the TBV to obtain a phenotype with the desired $h^{2}$. For Holstein and Australian Red individuals, breeds effects were sampled from $N(10,1)$ and $N(5,1)$, respectively, and added to their phenotypes.

\section{Statistical Analysis}

Breeding values were estimated for all scenarios using either GBLUP or a Bayesian method (Wang et al., 2016). The scenarios with 4,000 QTL, 30 chromosomes, and $\mathrm{h}^{2}$ of 0.6 in the $\mathrm{H}, \mathrm{HH}, \mathrm{J}, \mathrm{JJ}, \mathrm{R}$, and RR reference populations were also analyzed with a pedigree BLUP model.

GBLUP. The following model was fitted for GBLUP, using the ASReml software (Gilmour et al., 2008):

$$
\mathbf{y}=\mathbf{X b}+\mathbf{Z a}+\mathbf{G g}+\mathbf{e}
$$

where $\mathbf{y}$ is a vector of phenotypes, $\mathbf{X}, \mathbf{Z}$, and $\mathbf{G}$ are esign matrices allocating phenotypes to vectors $\mathbf{b}, \mathbf{a}$, and $\mathbf{g}$, with fixed effects (overall mean and breed), polygenic breeding values based on pedigree relationships, and genomic breeding values, respectively, and e is a vector of residual errors distributed as $N\left(0, \mathbf{E} \sigma_{e}^{2}\right)$, with identity matrix $\mathbf{E}$ and error variance $\sigma_{e}^{2}$. Polygenic and genomic breeding values were distributed as $N\left(0, \mathbf{A} \sigma_{a}^{2}\right)$ and $N\left(0, \mathbf{G R M} \sigma_{g}^{2}\right)$, respectively, where $\mathbf{A}$ is a numerator relationship matrix, $\sigma_{a}^{2}$ is the additive genetic variance not explained by genomic variants, GRM is a genomic relationship matrix, and $\sigma_{g}^{2}$ is the genetic variance explained by genomic variants. The GRM was constructed following Yang et al. (2011).

Bayes $\boldsymbol{R}$. We used the hybrid variant of the Bayes $\mathrm{R}$ model (Wang et al., 2016) that first uses an expectation-maximization (EM) module, followed by a Monte

Table 1. Composition of reference and validation populations

\begin{tabular}{|c|c|c|c|c|c|}
\hline \multirow{2}{*}{$\begin{array}{l}\text { Set } \\
\text { abbreviation }\end{array}$} & \multicolumn{5}{|c|}{ Number of individuals } \\
\hline & Total & Holstein & Jersey & Red Holstein & Australian Red \\
\hline$\overline{\mathrm{H}}$ & 7,989 & 7,989 & 0 & 0 & 0 \\
\hline $\mathrm{HH}$ & 15,547 & 15,547 & 0 & 0 & 0 \\
\hline $\mathrm{J}$ & 2,323 & 0 & 2,323 & 0 & 0 \\
\hline JJ & 4,310 & 0 & 4,310 & 0 & 0 \\
\hline $\mathrm{R}$ & 384 & 0 & 0 & 0 & 384 \\
\hline $\mathrm{RR}$ & 769 & 0 & 0 & 0 & 769 \\
\hline $\mathrm{H}+\mathrm{J}$ & 10,312 & 7,989 & 2,323 & 0 & 0 \\
\hline $\mathrm{H}+\mathrm{J}+\mathrm{RR}$ & 11,081 & 7,989 & 2,323 & 0 & 769 \\
\hline Validation & 2,117 & 582 & 551 & 870 & 114 \\
\hline
\end{tabular}


Table 2. Overview of simulated scenarios

\begin{tabular}{lrrrlll}
\hline nQTL $^{1}$ & $\mathrm{nS}^{2}$ & $\mathrm{nM}^{3}$ & $\mathrm{~nL}^{4}$ & $\mathrm{nChrs}^{5}$ & Reference population & Heritability $^{6}$ \\
\hline 100 & 0 & 0 & 100 & 30 & $\mathrm{H} / \mathrm{HH}$ & $0.3 / 0.6 / 0.9$ \\
2,000 & 1,360 & 615 & 25 & $12 / 30$ & $\mathrm{H} / \mathrm{HH}$ & $0.3 / 0.6 / 0.9$ \\
4,000 & 3,485 & 500 & 15 & $12 / 30$ & $\mathrm{H} / \mathrm{HH} / \mathrm{J} / \mathrm{JJ} / \mathrm{R} / \mathrm{RR} / \mathrm{H}+\mathrm{J} / \mathrm{H}+\mathrm{J}+\mathrm{RR}$ & $0.3 / 0.6 / 0.9$ \\
10,000 & 10,000 & 0 & 0 & 30 & $\mathrm{H} / \mathrm{HH}$ & $0.3 / 0.6 / 0.9$ \\
\hline
\end{tabular}

${ }^{1}$ Total number of QTL.

${ }^{2}$ Number of small QTL.

${ }^{3}$ Number of medium QTL.

${ }^{4}$ Number of large QTL.

${ }^{5}$ Number of chromosomes.

${ }^{6}$ See Table 1 for acronym definitions.

Carlo Markov chain (MCMC) model for 10,000 iterations, fitting the following model:

$$
\mathbf{y}=\mathbf{X b}+\mathbf{Z a}+\mathbf{W} \mathbf{v}+\mathbf{e},
$$

where $\mathbf{W}$ is a design matrix of genotypes and $\mathbf{v}$ a vector of variant effects, drawn from 4 normal distributions with $N\left(0,0 \sigma_{g}^{2}\right), \quad N\left(0,0.0001 \sigma_{g}^{2}\right), \quad N\left(0,0.001 \sigma_{g}^{2}\right), \quad$ and $N\left(0,0.01 \sigma_{g}^{2}\right)$, where $\sigma_{g}^{2}$ is the additive genetic variance

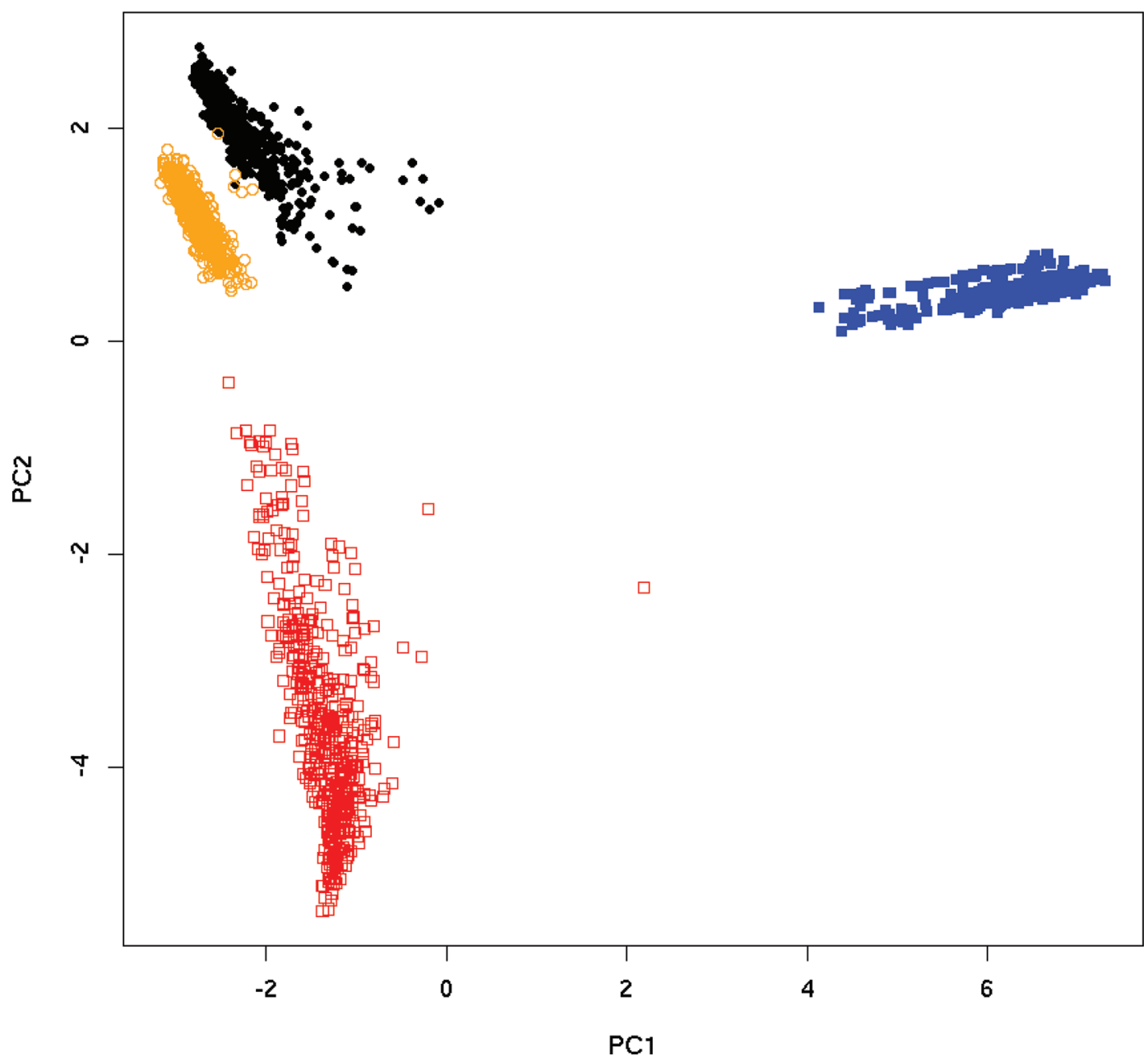

Figure 1. Principal component analysis of genomic relationships. First (x-axis) and second (y-axis) principal components of 500 randomly selected individuals for each population; black filled circles = Holstein; orange open circles = Red Holstein; blue filled squares $=$ Jersey; red open squares $=$ Australian Red. 
and prior distribution of the proportion of variants per distribution $\mathbf{P} \sim \operatorname{Dirichlet}(\boldsymbol{\alpha})$, with $\boldsymbol{\alpha}=[1,1,1,1]$.

Pedigree BLUP. The pedigree BLUP model was similar to the GBLUP model, except for the omission of the $\mathbf{G g}$ term:

$$
\mathbf{y}=\mathbf{X b}+\mathbf{Z a}+\mathbf{e} .
$$

Accuracy Obtained by Cross-Validation. The accuracy was empirically estimated as the correlation between GEBV estimated by GBLUP or Bayes R, using the estimates of both the marker effects and the polygenic term, and the simulated TBV of animals not included in the reference population.

Deterministic Accuracy of Genomic Prediction. We used the following formula as the basis for computing the prediction accuracy a priori (Goddard et al., 2011):

$$
r=\sqrt{\frac{\theta}{1+\theta-r^{2} h^{2}}},
$$

where $\theta=\frac{T h^{2}}{M_{e}}, T$ is the size of the reference population, and $M_{e}$ is the effective number of independent chromosome segments; $r$ was estimated by solving Equation 1 for $r$ using the solve() command in R Core Team (2015). For within-breed prediction, $M_{e}$ was estimated as

$$
M_{e}=\frac{1}{\operatorname{Var}\left(\mathbf{G}_{i j}-\mathbf{A}_{i j}\right)},
$$

where $\mathbf{G}_{i j}$ and $\mathbf{A}_{i j}$ are the genomic and pedigree relationships, respectively, between individuals $i$ and $j(i \neq$ $j$ ), and the variance of all $i j$ pairs in the population was used to estimate $M_{e}$. Both $\mathbf{G}$ and $\mathbf{A}$ were constructed using only the animals of the breed for which $M_{e}$ was estimated. For across-breed prediction, G was constructed using only the reference animals of 1 breed and the validation animals of the other breed and genotypes were standardized based on the overall allele frequencies of all animals included in the analysis or breedspecific allele frequencies. Subsequently, the acrossbreed $M_{e}$ was estimated only based on the relationships between the reference and validation populations, following Wientjes et al. (2015b). Because pedigree relationships between breeds were 0 , only genomic relationships were used to estimate $M_{e}$ across breeds as $M_{e}=\frac{1}{\operatorname{Var}\left(\mathbf{G}_{i j}\right)}$.
Alternatively, we used the accuracies obtained by cross-validation and Equation 1 to estimate $\theta$, using the nonlinear least squares (nls) function in $\mathrm{R}$ Core Team (2015). Subsequently, $\theta$ was then used to obtain $M_{e}$, by $M_{e}=\frac{T h^{2}}{\theta}$, and compared with $M_{e}$ computed using Equation 2. For every reference population, the $\theta$ value was calculated independently for each of the 4 different validation sets.

In statistical theory, if 2 sources of information with independent sampling errors are combined, Fisher's information equals the sum of the information coming from each of the sources (which is depicted by $\theta$ here) and the accuracy can be predicted from this summed information (i.e., the sum of the $\theta$ from each source). Following from this principle and from Equation 1, doubling the size of the reference population should result in doubling $\theta$. We tested whether doubling $\theta$ correctly estimated the accuracy when the size of the reference population was doubled. For example, if we calculate the realized accuracies obtained by cross-validation from the $\mathrm{H}, \mathrm{J}$, or $\mathrm{R}$ reference population and then solve for $\theta$ using Equation 1 (we call this $\theta_{I}$ ), the accuracy using the HH, JJ, or RR reference population $\left(r_{I I}\right)$ was predicted a priori as

$$
r_{I I}=\sqrt{\frac{2 \theta_{I}}{1+2 \theta_{I}-r^{2} h^{2}}} .
$$

However, the 2 half reference populations do not provide completely independent information because animals of the same breeds are sometimes highly related. To account for this, we investigated whether removing the pedigree component from $\theta$ would result in a more accurate prediction of the accuracy. First, for the $\mathrm{H}$, $\mathrm{J}$, and $\mathrm{R}$ reference sets, $\theta_{I, P}$ was estimated, by solving for $\theta$ using Equation 1 with the realized accuracies of cross-validation for pedigree BLUP. Subtracting this from $\theta_{I}$ leaves the part of $\theta_{I}$ that can be attributed to the genomic markers rather than pedigree information; that is, $\theta_{I, G}=\theta_{I}-\theta_{I, P}$. To account for the contribution of pedigree information to $\theta_{I I}$ (i.e., the $\theta$ used to predict the accuracy of the $\mathrm{HH}$, JJ, and RR reference sets), $\theta_{I I, P}$ was estimated using Equation 1 with the accuracies obtained by cross-validation for pedigree BLUP for the HH, JJ, and RR reference sets. The accuracy of genomic prediction was then estimated as

$$
r=\sqrt{\frac{2 \theta_{I, G}+\theta_{I I, P}}{1+2 \theta_{I, G}+\theta_{I I, P}-r_{I I}^{2} h_{2}}} .
$$


In the multibreed reference populations, $\mathrm{H}+\mathrm{J}$ and $\mathrm{H}+\mathrm{J}+\mathrm{RR}$, we investigated whether adding up $\theta$ obtained for each of the validation populations using the $\mathrm{H}, \mathrm{J}$, and RR reference populations could be used to estimate the accuracy of the multibreed reference set for each of the breeds in the validation population:

$$
r_{H+J \rightarrow V}=\sqrt{\frac{\theta_{H \rightarrow V}+\theta_{J \rightarrow V}}{1+\theta_{H \rightarrow V}+\theta_{J \rightarrow V}-r_{H+J \rightarrow V}^{2} h^{2}}},
$$

and

$$
r_{H+J+R R \rightarrow V}=\sqrt{\frac{\theta_{H \rightarrow V}+\theta_{J \rightarrow V}+\theta_{R R \rightarrow V}}{1+\theta_{H \rightarrow V}+\theta_{J \rightarrow V}+\theta_{R R \rightarrow V}-r_{H+J+R R \rightarrow V}^{2} h^{2}}},
$$

where $\theta_{H \rightarrow V}, \theta_{J \rightarrow V}$, and $\theta_{R R \rightarrow V}$ were estimated using Equation 1 with the accuracies obtained by cross-validation for validation population $V$ ( $V=$ Holstein, Jersey, Red Holstein, or Australian Red) for the H, J, and RR reference populations, respectively; and $r_{H+J \rightarrow V}$ and $r_{H+J+R R \rightarrow V}$ are the accuracies for validation population $V$ using the $\mathrm{H}+\mathrm{J}$ and $\mathrm{H}+\mathrm{J}+\mathrm{RR}$ reference populations, respectively. We also applied this theory to a real data set, using $\mathrm{h}^{2}$ and accuracies published by Kemper et al. (2015) that used a multibreed reference set containing 11,527 Holstein and 4,687 Jersey animals to predict GEBV for milk traits in validation populations containing 251 Holstein, 81 Jersey, and 361 Australian Red individuals. For all milk traits, a heritability of 0.33 was used, as reported by Kemper et al. (2015).

We compared the increase in accuracy obtained by cross-validation when the heritability was increased from 0.3 to 0.6 or 0.9 , with the predicted increase using Equation 1:

$$
r_{0.6}=\sqrt{\frac{2 \theta_{0.3}}{1+2 \theta_{0.3}-r_{0.6}^{2} h^{2}}}
$$

and

$$
r_{0.9}=\sqrt{\frac{3 \theta_{0.3}}{1+3 \theta_{0.3}-r_{0.9}^{2} h^{2}}},
$$

where $\theta_{0.3}$ was estimated using Equation 1 and the accuracy obtained by cross-validation for the scenario with a heritability of 0.6 , and $r_{0.6}$ and $r_{0.9}$ were the accuracies for the scenarios with heritabilities of 0.6 and 0.9, respectively. Alternatively, we used the accuracies obtained by pedigree BLUP to obtain $\theta_{0.3, P}$ for the scenarios with a heritability of 0.3 and used those to obtain $\theta_{0.3, G}=\theta_{0.3}-\theta_{0.3, P}$. The accuracies were then estimated as

$$
r_{0.6}=\sqrt{\frac{2 \theta_{0.3, G}+\theta_{0.6, P}}{1+2 \theta_{0.3, G}+\theta_{0.6, P}-r_{0.6}^{2} h^{2}}},
$$

and

$$
r_{0.9}=\sqrt{\frac{3 \theta_{0.3, G}+\theta_{0.9, P}}{1+3 \theta_{0.3, G}+\theta_{0.9, P}-r_{0.9}^{2} h^{2}}},
$$

where $\theta_{0.6, P}$ and $\theta_{0.9, P}$ were obtained using Equation 1 and the accuracies obtained by cross-validation with pedigree BLUP for the scenarios with a heritability of 0.6 and 0.9 , respectively.

\section{RESULTS}

\section{Using the GRM to Predict $M_{\mathrm{e}}$}

Estimating the prediction accuracy using $M_{e}$ from the GRM resulted in an overestimation of the accuracy obtained by cross-validation, as shown in Figure 2. This is equivalent to saying that $M_{e}$ estimated from the GRM was much less than $M_{e}$ derived from the accuracies of Bayes R and GBLUP (Table 3) in almost all scenarios. This discrepancy could be due to close relationships among some of the reference animals, which leads to a low value of $M_{e}$ from the GRM. For across-breed prediction, using breed-specific allele frequencies to construct the GRM that was used to estimate $M_{e}$ resulted in an increase in $M_{e}$, thus reducing the overestimation of the prediction accuracy. Although the $M_{e}$ estimated using breed-specific allele frequencies was reasonably close to $M_{e}$ derived from Bayes R, it was substantially lower than $M_{e}$ derived from GBLUP. Therefore, in the rest of the study, we did not use the GRM to estimate $M_{e}$, but instead used realized accuracy from a small, singlebreed reference population to calculate $\theta$ and then combined these $\theta$ values to predict the accuracy expected from larger or multibreed reference populations.

\section{Reference Population Size}

Table 4 compares the accuracy predicted using $\theta=$ $2 \theta_{I}$ or $\theta=2 \theta_{I, G}+\theta_{I I, P}$, with the accuracy obtained by cross-validation when the size of the single-breed reference population doubled. In all breeds, using $\theta=2 \theta_{I}$ slightly overpredicted the accuracy when Bayes $\mathrm{R}$ was used. For GBLUP, the accuracy was underpredicted for Holstein and Jersey, but overpredicted for Australian Red. Removing the pedigree information, by using $\theta=$ 
H

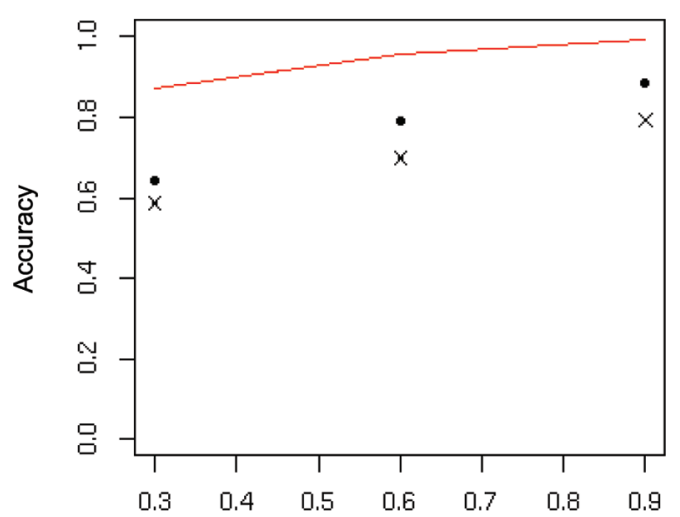

J

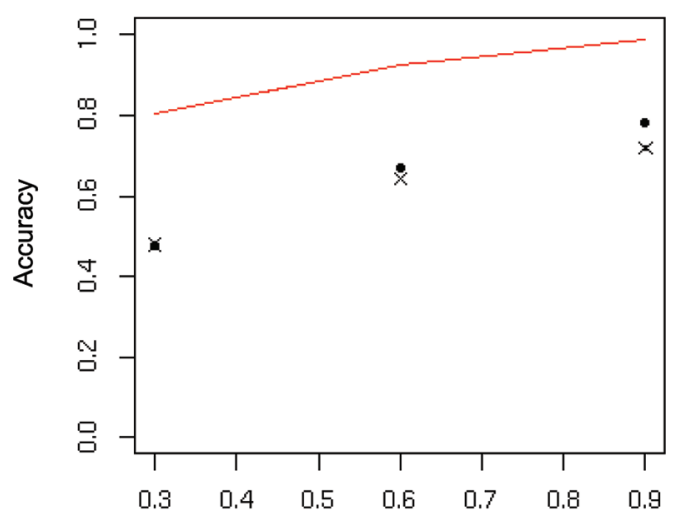

R

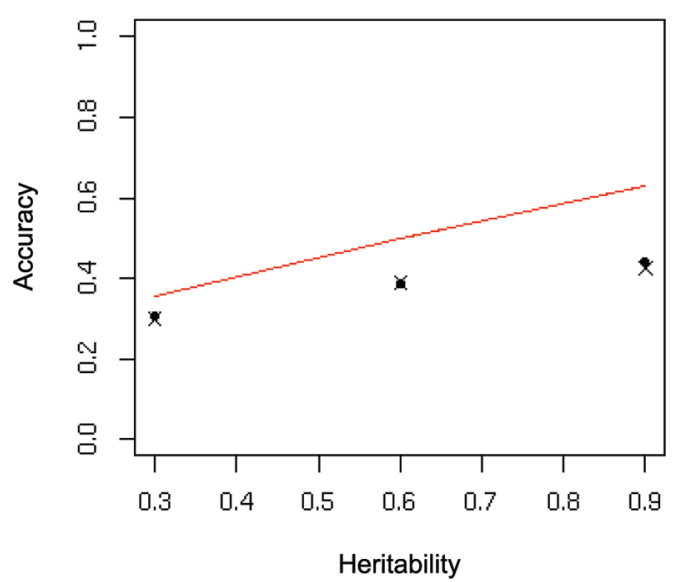

HH

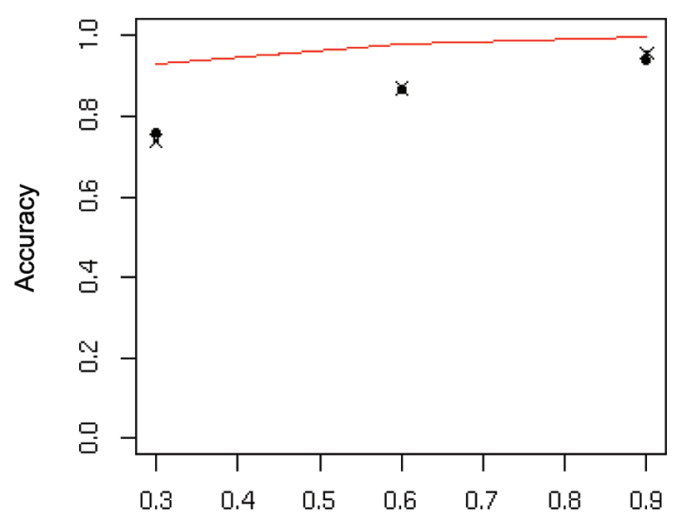

JJ

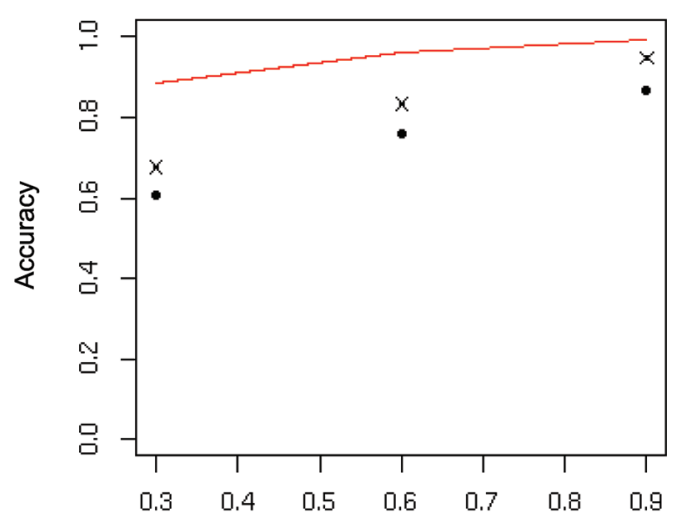

RR

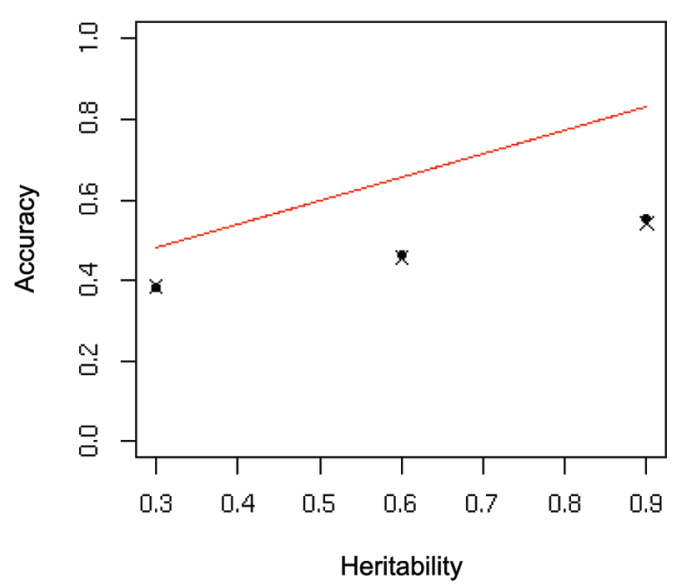

Figure 2. Comparison accuracies obtained by cross-validation and accuracies estimated using deterministic formulae for within-breed prediction. Accuracies obtained by cross-validation for Bayes R (dots), genomic BLUP $(\times$ ), and based on Equation 3 (red line) using the size of the reference population, heritability, and number of independent chromosomal segments $\left(M_{e}\right)$ estimated from the genomic and pedigree relationship matrices, for scenarios with 4,000 QTL and 30 chromosomes and a reference population containing 7,989 Holstein (H), 15,547 Holstein (HH), 2,323 Jersey (J), 4,310 Jersey (JJ), 348 Australian Red (R), or 769 Australian Red (RR) individuals. 
Table 3. Effective number of chromosome segments $\left(M_{e}\right)$ estimated using realized accuracies from Bayes R and genomic BLUP or using the genomic relationship matrix $(\mathrm{GRM})$

\begin{tabular}{|c|c|c|c|c|c|c|c|}
\hline Reference & Validation & $\mathrm{nChrs}^{1}$ & $\mathrm{nQTL}^{2}$ & \multicolumn{3}{|c|}{$M_{e}$} & GRM-BS $^{4}$ \\
\hline Holstein & Holstein & 12 & 2,000 & 2,546 & 4,054 & 839 & - \\
\hline Holstein & Holstein & 30 & 2,000 & 3,218 & 5,541 & 997 & - \\
\hline Holstein & Holstein & 12 & 4,000 & 3,669 & 4,344 & 839 & - \\
\hline Holstein & Holstein & 30 & 4,000 & 4,644 & 6,134 & 997 & - \\
\hline AusRed $^{5}$ & AusRed & 30 & 4,000 & 1,765 & 1,833 & 816 & - \\
\hline Holstein & $\mathrm{RedHol}^{6}$ & 30 & 4,000 & 6,904 & 12,509 & 2,726 & 4,375 \\
\hline Holstein & Jersey & 30 & 4,000 & 17,358 & 69,759 & 920 & 15,397 \\
\hline Holstein & AusRed & 30 & 4,000 & 14,636 & 58,071 & 2,101 & 18,547 \\
\hline
\end{tabular}

${ }^{1}$ Number of chromosomes.

${ }^{2}$ Number of QTL.

${ }^{3}$ If reference and validation are the same breed, $M_{e}$ was estimated based on genomic and pedigree relationships. If reference and validation population are different breeds, $M_{e}$ was estimated based on relationships between reference and validation populations using genotypes standardized using across-breed allele frequencies.

${ }^{4} M_{e}$ for across-breed prediction estimated based on relationships between reference and validation populations using genotypes standardized using breed-specific allele frequencies.

${ }^{5}$ Australian Red.

${ }^{6}$ Red Holstein.

$2 \theta_{I, G}+\theta_{I I, P}$ to predict the accuracy, further increased overestimation in Holstein and Jersey when Bayes $\mathrm{R}$ was used and resulted in an overestimation in all breeds when GBLUP was used. Accuracies predicted using the accuracies obtained by cross-validations in the smaller reference population were closer to the realized accuracies than when the GRM was used to estimate $M_{e}$ and, subsequently, the accuracy.

\section{Use of a Reference Population from a Breed Different from the Validation Population}

Figures 3 and 4 show the cross-validation accuracy for different reference populations using Bayes $\mathrm{R}$ and GBLUP, respectively, for scenarios with 4,000 QTL, a heritability of 0.6 , using 30 chromosomes. The heritabilities estimated with GBLUP within each reference populations can be found in Appendix Table A2.

The accuracy was largest when the reference population contained animals of the same breed as the validation population and increased when the size of the reference population increased. For within-breed prediction, although Bayes $\mathrm{R}$ usually gave higher accuracy than GBLUP, the differences in accuracies were small. However, when the reference and validation populations were of different breeds, the advantage of Bayes R over GBLUP was larger and more consistent. For instance, predicting Australian Red from the $\mathrm{HH}$ reference population resulted in an accuracy of 0.69 when Bayes $R$ was used, but only 0.38 with GBLUP.

Table 4. Within-breed prediction accuracy and SE when the size of the reference population is doubled ${ }^{1}$

\begin{tabular}{llccc}
\hline Method & Accuracy & Holstein & Jersey & AusRed $^{2}$ \\
\hline Bayes R & Cross-validation & $0.87 \pm 0.01$ & $0.76 \pm 0.02$ & $0.47 \pm 0.04$ \\
& $2 \theta_{I}{ }^{3}$ & $0.89 \pm 0.01$ & $0.80 \pm 0.02$ & $0.52 \pm 0.04$ \\
\multirow{3}{*}{ GBLUP } & $2 \theta_{I, G}+\theta_{I I, P}{ }^{4}$ & $0.92 \pm 0.00$ & $0.88 \pm 0.01$ & $0.50 \pm 0.04$ \\
& Cross-validation & $0.87 \pm 0.01$ & $0.83 \pm 0.01$ & $0.46 \pm 0.05$ \\
& $2 \theta_{I}$ & $0.83 \pm 0.00$ & $0.78 \pm 0.01$ & $0.53 \pm 0.04$ \\
& $2 \theta_{I, G}+\theta_{I I, P}$ & $0.90 \pm 0.00$ & $0.87 \pm 0.00$ & $0.51 \pm 0.04$ \\
\hline
\end{tabular}

${ }^{1}$ Deterministic predictions are compared with cross-validation accuracies, scenario with a heritability of 0.6 and 4,000 QTL. ${ }^{2}$ Australian Red.

${ }^{3}$ Accuracy estimated as $r=\sqrt{\frac{\theta}{1+\theta-r^{2} h^{2}}}$, where $\theta=2 \theta_{I}$.

${ }^{4}$ Accuracy estimated as $r=\sqrt{\frac{\theta}{1+\theta-r^{2} h^{2}}}$, where $\theta=2 \theta_{I, G}+\theta_{I I, P}$. 
Holstein
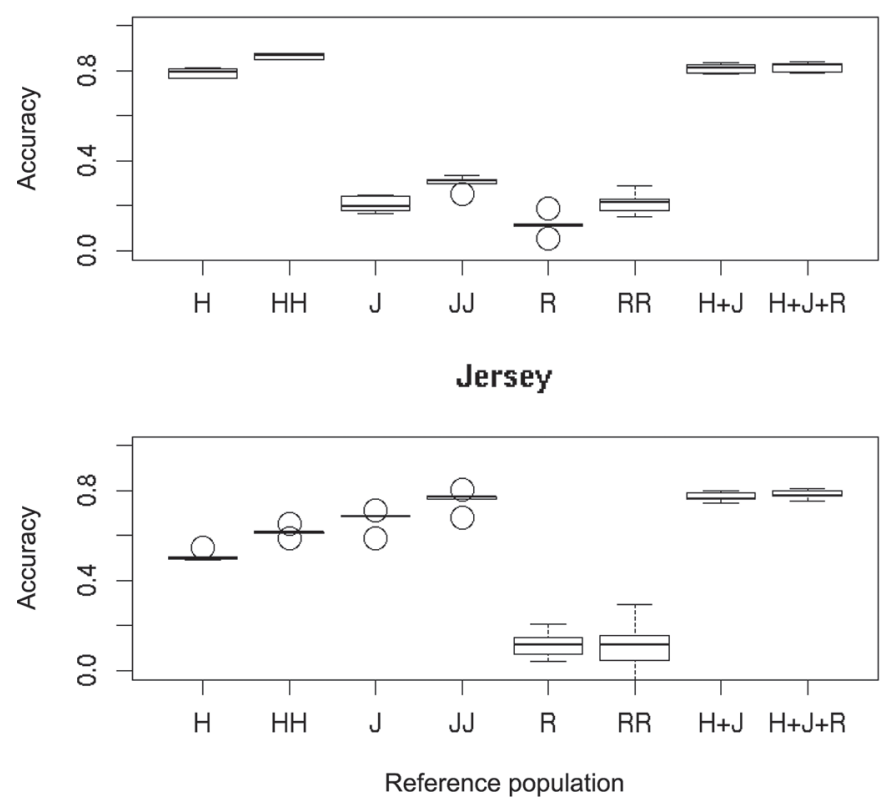

Red Holstein

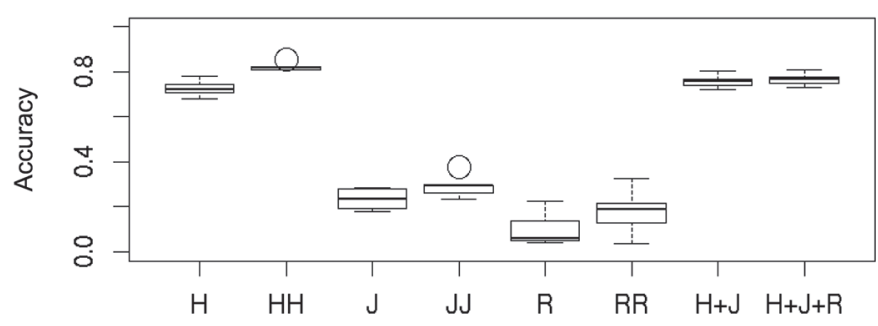

Australian Red

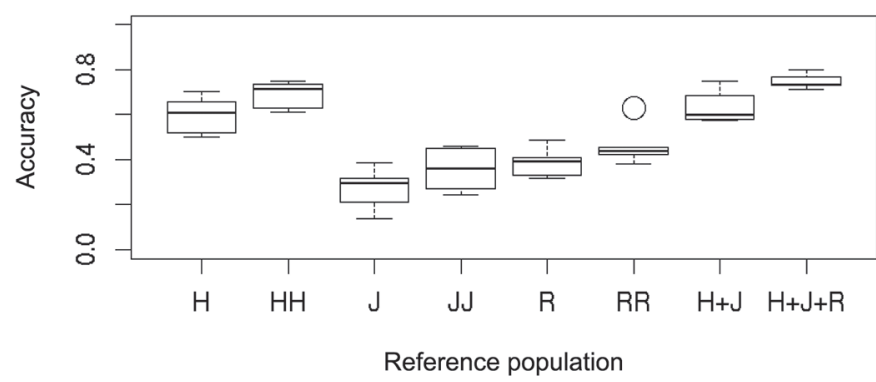

Figure 3. Influence of the composition of the reference population on prediction accuracy of Bayes R. For scenarios with a heritability of 0.6, 4,000 QTL, and 30 chromosomes, the reference population contained 7,989 Holstein (H), 15,547 Holstein (HH), 2,323 Jersey (J), 4,310 Jersey (JJ), 348 Australian Red (R), 769 Australian Red (RR), 7,989 Holstein and 2,323 Jersey (H+J) or 7,989 Holstein, 2,323 Jersey, and 769 Australian Red $(\mathrm{H}+\mathrm{J}+\mathrm{RR})$ individuals. The boxes show the middle $50 \%$ of values, the line shows the median, the whiskers show values outside the middle $50 \%$, and the circles are outliers.

Furthermore, we found more advantage for Bayes $\mathrm{R}$ compared with GBLUP when a reference of mixed breeds was used. In particular, when the single-breed reference was small ( $\mathrm{J}$ or $\mathrm{R}$ ), the mixed-breed reference gave a significant advantage when using Bayes $\mathrm{R}$ but little or no advantage for GBLUP.

Table 5 compares accuracies for multibreed prediction calculated a priori using Equation 5 with those obtained by cross-validation. For all breeds and models, accuracies predicted using Equation 5 were within 0.03 of the accuracy obtained by cross-validation. The accuracy with Bayes $\mathrm{R}$ tended to be slightly underestimated, whereas the accuracy with GBLUP tended to be slightly overestimated. As shown in Table 6, multibreed accuracies in a real data set were reasonably well predicted for the Holstein and Jersey validation sets, with up to 0.03 differences between the accuracy predicted using Equation 5 and that obtained by crossvalidation. Predicting Australian Red resulted in larger deviations, up to 0.13 for Bayes $\mathrm{R}$ and up to 0.07 for GBLUP.

Appendix Tables A3, A4, A5, and A6 compare the ranking of GEBV when different reference populations were used. Using different reference populations changed the rankings, with the largest differences occurring between reference populations that resulted in the largest differences in accuracy.

\section{Heritability}

Figure 5 shows the effect of heritability on realized prediction accuracy for scenarios with 4,000 QTL using the $\mathrm{H}$ reference population and 30 chromosomes. When the heritability increased, the accuracy increased in all breeds and for both methods. As with all levels of heritability, Bayes $\mathrm{R}$ resulted in a higher accuracy than GBLUP, and this superiority increased both when the heritability increased and the genetic relatedness between the reference and validation population decreased. For example, the difference between Bayes R and GBLUP was 0.05 and 0.09 with heritabilities of 0.3 and 0.9 when predicting within Holstein and 0.16 and 0.31 when predicting Australian Red, respectively.

As shown in Table 7, using $\theta=2 \theta_{0.3}$ to predict the accuracy for scenarios with $\mathrm{h}^{2}=0.6$ resulted in a slight over- or underestimation of the accuracy, depending on breed and method. The largest deviation, 0.04, was found in Australian Red using Bayes R. This deviation was further reduced by applying a correction for the pedigree relationships, using $\theta=2 \theta_{0.3, G}+\theta_{0.6, P}$, for both Bayes R and GBLUP. Predicting the accuracy for the scenarios with a heritability of 0.9 resulted in larger deviations and overestimated the accuracy, regardless of breeds and method. The largest deviation was found when $\theta=3 \theta_{0.3}$ was used to predict the accuracy of 
Holstein
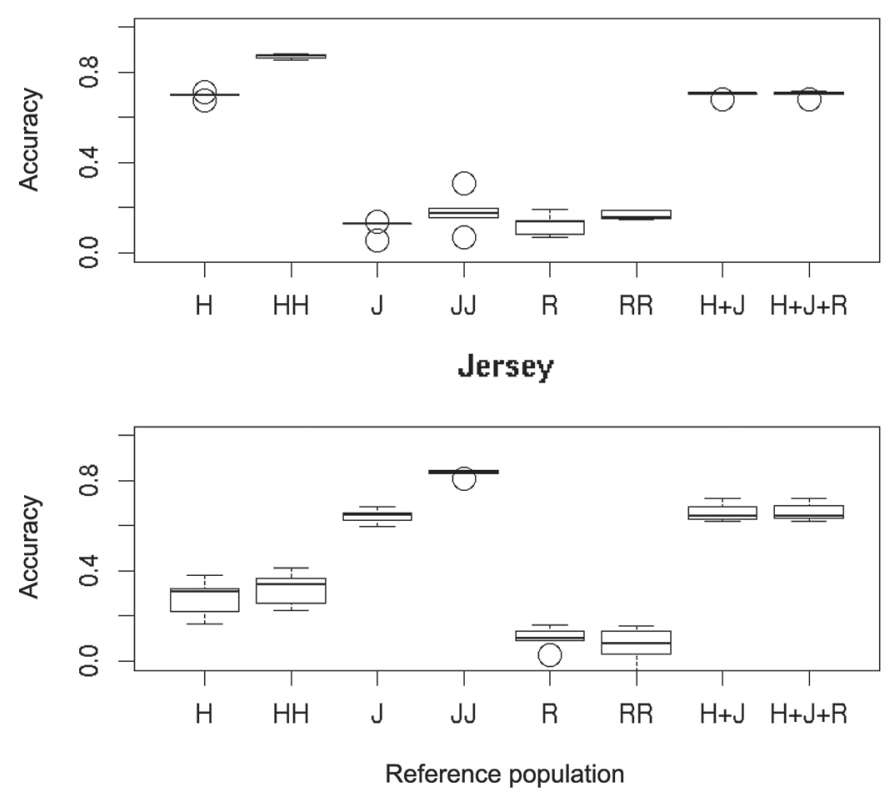

Red Holstein
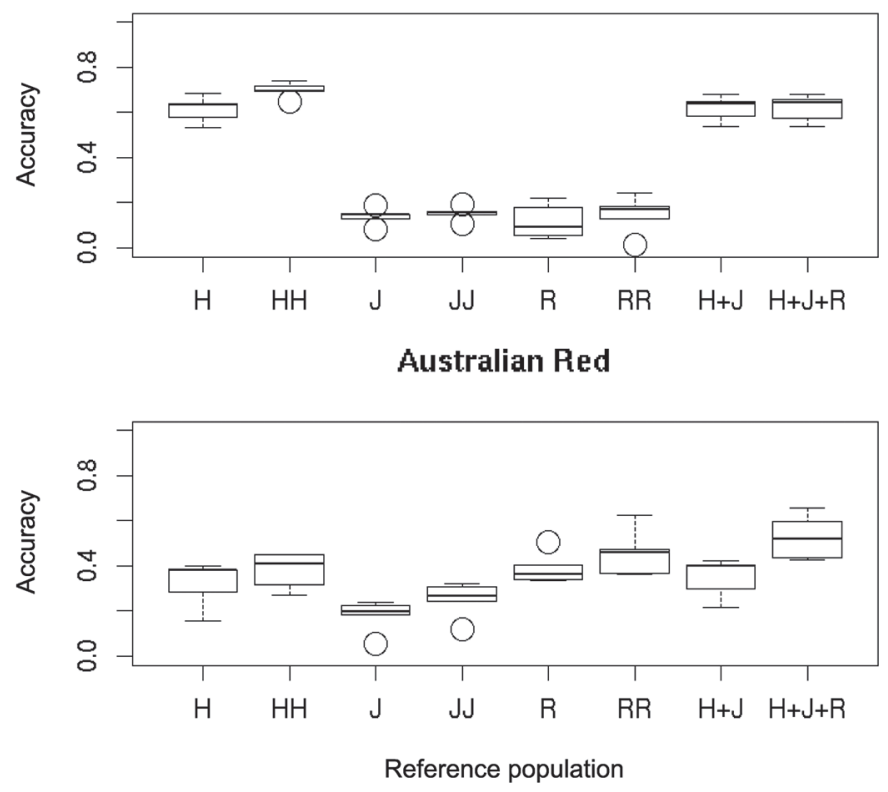

Figure 4. Influence of the composition of the reference population on prediction accuracy of genomic BLUP. For scenarios with a heritability of 0.6, 4,000 QTL, and 30 chromosomes, the reference population contained 7,989 Holstein (H), 15,547 Holstein (HH), 2,323 Jersey (J), 4,310 Jersey (JJ), 348 Australian Red (R), 769 Australian Red (RR), 7,989 Holstein and 2,323 Jersey (H+J), or 7,989 Holstein, 2,323 Jersey, and 769 Australian Red $(\mathrm{H}+\mathrm{J}+\mathrm{RR})$ individuals. The boxes show the middle $50 \%$ of values, the line shows the median, the whiskers show values outside the middle $50 \%$, and the circles are outliers.

Australian Red using Bayes R, 0.10 higher than the accuracy obtained by cross-validation. Using $\theta=3 \theta_{0.3, G}$ $+\theta_{0.9, P}$ to predict the accuracy reduced the overestimation for all breeds and methods.

\section{Size of the Genome}

Table 8 shows the influence of the number of chromosomes on the prediction accuracy for scenarios with a heritability of 0.6 using the $\mathrm{H}$ reference population. Reducing the number of chromosomes resulted in an increase in accuracy for most scenarios, especially using GBLUP. Averaged across breeds and number of QTL, the accuracy in the scenarios with 12 chromosomes was 0.005 and 0.056 larger than the accuracy in the scenarios with 30 chromosomes using Bayes R and GBLUP, respectively. Generally, differences between the scenarios with 12 and 30 chromosomes were larger for GBLUP than for Bayes R.

\section{The Number of QTL and Genetic Architecture}

Increasing the number of QTL resulted in a decrease in accuracy in Bayes $\mathrm{R}$ but had no effect on the accuracy of GBLUP, as shown in Figure 6 and 7. For this comparison, the reference population was $\mathrm{H}$ and the heritability was 0.6. Differences were more pronounced for across-breed prediction than within-breed prediction. For example, with 100 QTL drawn from a single normal distribution, predicting Jersey breeding values from the $\mathrm{H}$ reference population resulted in an accuracy of 0.94 when Bayes $\mathrm{R}$ was used, but only 0.25 when GBLUP was used. In the scenario with 10,000 QTL drawn from a single normal distribution, accuracies obtained for Jersey were 0.27 and 0.30 for Bayes R and GBLUP, respectively.

\section{DISCUSSION}

\section{Predicting $M_{\mathrm{e}}$ from the GRM}

Using the GRM to predict $M_{e}$ and Equation 1 to predict accuracy resulted in an overestimation of accuracy compared with cross-validation for most scenarios. Likewise, the GRM implied much lower values of $M_{e}$ than obtained from the cross-validation accuracy (Table 3). The underestimation of $M_{e}$ from the GRM probably occurred because a small number of pairs of animals are closely related. These pairs have a much higher relationship to each other than usual and, consequently, lead to a high variance of the elements of the GRM and thus a low estimate of $M_{e}$. These high relationships within a breed occur due to pedigree relationships (known and unknown) and between breeds 
Table 5. Multibreed prediction accuracy for 4 validation breeds using the simulated data set ${ }^{1}$

\begin{tabular}{|c|c|c|c|c|c|c|}
\hline refPop ${ }^{2}$ & Method & Accuracy & Holstein & $\operatorname{RedHol}^{3}$ & Jersey & AusRed $^{4}$ \\
\hline \multirow{2}{*}{$\mathrm{H}+\mathrm{J}^{5}$} & & Equation $5^{6}$ & $0.80 \pm 0.01$ & $0.74 \pm 0.02$ & $0.76 \pm 0.01$ & $0.64 \pm 0.04$ \\
\hline & Genomic BLUP & Cross-validation & $0.70 \pm 0.01$ & $0.62 \pm 0.03$ & $0.66 \pm 0.02$ & $0.34 \pm 0.04$ \\
\hline \multirow{3}{*}{$\mathrm{H}+\mathrm{J}+\mathrm{RR}^{7}$} & BayesR & Cross-validation & $0.82 \pm 0.01$ & $0.77 \pm 0.01$ & $0.78 \pm 0.01$ & $0.75 \pm 0.02$ \\
\hline & & Equation $5^{8}$ & $0.81 \pm 0.01$ & $0.75 \pm 0.02$ & $0.77 \pm 0.01$ & $0.72 \pm 0.03$ \\
\hline & Genomic BLUP & Cross-validation & $0.70 \pm 0.01$ & $0.62 \pm 0.03$ & $0.66 \pm 0.02$ & $0.53 \pm 0.04$ \\
\hline
\end{tabular}

${ }^{1}$ Deterministic predictions are compared with cross-validation accuracies, scenario with a heritability of 0.6 and 4,000 QTL.

${ }^{2}$ Reference population.

${ }^{3}$ Red Holstein.

${ }^{4}$ Australian Red.

${ }^{5}$ Holstein and Jersey.

${ }^{6}$ Accuracy for the validation population $(V)$ estimated using equation $5: r_{H+J \rightarrow V}=\sqrt{\frac{\theta_{H \rightarrow V}+\theta_{J \rightarrow V}}{1+\theta_{H \rightarrow V}+\theta_{J \rightarrow V}+\theta_{R R \rightarrow}-r_{H+J \rightarrow V}^{2} h^{2}}}$.
${ }^{7}$ Holstein, Jersey, and Australian Red.

${ }^{8}$ Accuracy estimated using equation $5: r_{H+J+R R \rightarrow V}=\sqrt{\frac{\theta_{H \rightarrow V}+\theta_{J \rightarrow V}+\theta_{R R \rightarrow V}}{1+\theta_{H \rightarrow V}+\theta_{J \rightarrow V}+\theta_{R R \rightarrow V}-r_{H+J+R R \rightarrow V}^{2} h^{2}}}$.

due to crossbreeding (e.g., grading up from Jersey to Holstein). Consequently, in these data, the GRM is not useful for predicting accuracy of GEBV. Wientjes et al. (2013) reported an $M_{e}$ of 837 estimated from genomic relationships between highly related Holstein-Friesian individuals, within the range of the estimates obtained for our within-breed scenarios using the GRM. Erbe et al. (2013) estimated $M_{e}$ based on accuracies obtained by cross-validation, comparing various formulae. Their estimates of $M_{e}$ using a Holstein-Friesian data were lower than the estimates of $M_{e}$ we obtained based on cross-validation accuracies of either GBLUP or Bayes $\mathrm{R}$, varying between 900 and 2,800 depending on the trait and formula that was used to estimate $M_{\mathrm{e}}$, and between 150 to 400 for a Brown-Swiss data set. The differences in $M_{e}$ obtained by our study and Erbe et al. (2013) could be due to differences between the populations used.

Brard and Ricard (2015) showed that differences in method used to estimate $M_{e}$ can lead to large differences in the estimate of $M_{e}$, making it difficult to use formulae relying on an $M_{e}$ estimate in practice. Therefore, they suggested to first estimate $M_{e}$ in a population where genomic prediction accuracies are available and use those estimates to predict the accuracy in similar populations. We also found that this was the best approach.

In simulation studies, Wientjes et al. (2015b) and Vandenplas et al. (2017) computed $M_{e}$ based on the difference between pedigree and genomic prediction

Table 6. Predicting accuracies for a real multibreed Holstein and Jersey reference population compared with deterministic predictions

\begin{tabular}{|c|c|c|c|c|c|c|c|}
\hline valPop $^{1}$ & Method & Accuracy & Fat & Milk & Prot & Fat $\%^{2}$ & $\operatorname{Prot} \%^{3}$ \\
\hline Holstein & & Equation $5^{4}$ & 0.64 & 0.64 & 0.61 & 0.83 & 0.83 \\
\hline \multirow[t]{3}{*}{ Jersey } & BayesR & Cross-validation & 0.56 & 0.69 & 0.71 & 0.76 & 0.79 \\
\hline & & Equation 5 & 0.56 & 0.72 & 0.73 & 0.79 & 0.81 \\
\hline & Genomic BLUP & Cross-validation & 0.58 & 0.64 & 0.69 & 0.66 & 0.77 \\
\hline \multirow{3}{*}{ AusRed $^{5}$} & & Equation 5 & 0.39 & 0.21 & 0.19 & 0.61 & 0.48 \\
\hline & Genomic BLUP & Cross-validation & 0.17 & 0.11 & 0.04 & 0.46 & 0.48 \\
\hline & & Equation 5 & 0.17 & 0.10 & 0.11 & 0.45 & 0.51 \\
\hline
\end{tabular}

\footnotetext{
${ }^{1}$ Validation population.

${ }^{2}$ Fat percentage.

${ }^{3}$ Protein percentage.

${ }^{4}$ Accuracy for the validation population $(V)$ estimated using equation 5: $r_{H+J \rightarrow V}=\sqrt{\frac{\theta_{H \rightarrow V}+\theta_{J \rightarrow V}}{1+\theta_{H \rightarrow V}+\theta_{J \rightarrow V}-r_{H+J \rightarrow V}^{2} h^{2}}}$.
${ }^{5}$ Australian Red.
} 
relationships. Using realized genotypes of dairy cattle populations and simulated phenotypes, Wientjes et al. (2015b) used $M_{e}$ estimates based on genomic and pedigree relationships between the reference and validation populations as parameters in a deterministic formula used to estimate prediction accuracy. Similar to our results, Wientjes et al. (2015b) obtained an overestimation of the accuracy and argued that this could be because the prediction markers used may not capture all genetic variance. However, this cannot be the explanation for our simulation experiment, because all QTL were included among the markers and the markers should have been able to capture all the genetic variance. In a simulation study mimicking cross-breeding, as is common in pigs and chicken breeding programmes, Vandenplas et al. (2017) used formulae similar to Wientjes et al. (2015b) to estimate prediction accuracy and stated that deviations from the accuracies obtained by cross-validation were likely due to inaccuracies in the estimation of $M_{e}$.

\section{Holstein}
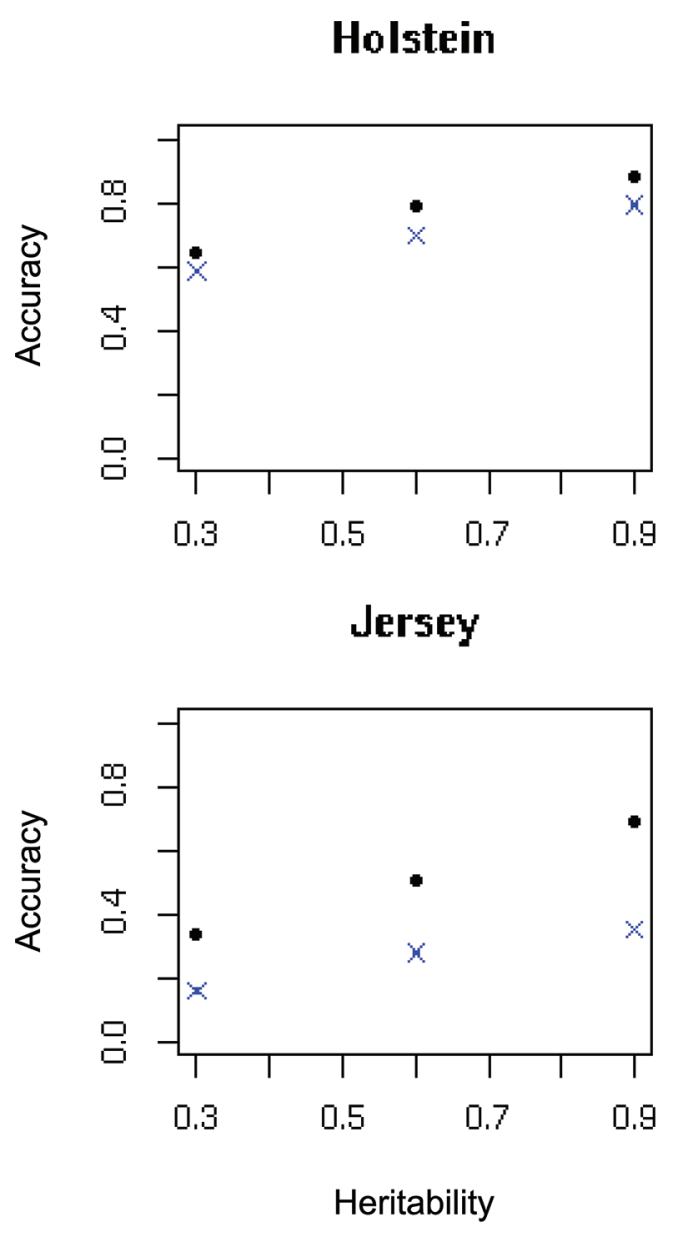

Misztal (2016) and Pocrnic et al. (2016a,b) propose that the number of animals required to explain almost all GRM variance equals $M_{e}$ and can be estimated as the number of largest Eigen values explaining nearly all variance. Pocrnic et al. (2016b) applied this to Holstein and Jersey cattle populations, where they required more than 10,000 Eigen values to explain 98\% of the variance. This number was substantially larger than values for $M_{e}$ obtained in our study by either the GRM or cross-validation and would have resulted in an underestimation of the prediction accuracy. Our results agreed with the literature that estimating $M_{e}$ from the genotype data alone (e.g., from the GRM) is not a satisfactory method to predict accuracy.

\section{Combining Reference Populations and the Number of QTL}

The size and composition of the reference population had a large effect on the prediction accuracy of both

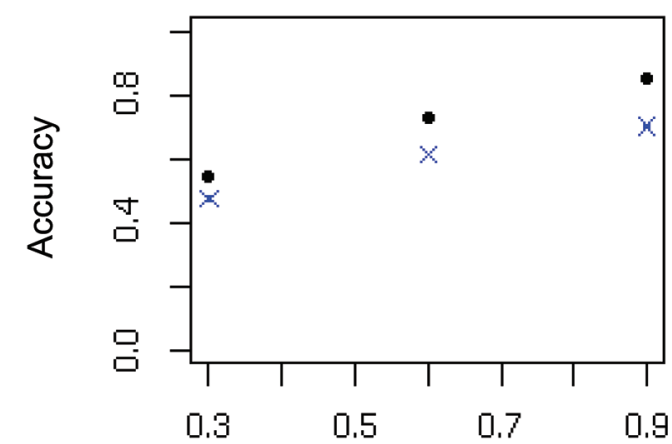

Australian Red

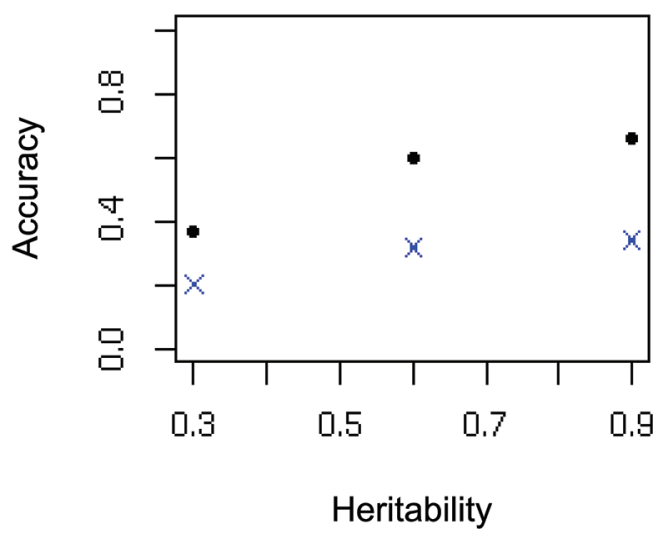

Figure 5. Influence of the heritability on prediction accuracy for scenarios with 4,000 QTL, 30 chromosomes, and a reference population containing 7,989 Holstein individuals; dots $=$ Bayes $\mathrm{R}, \times=$ genomic BLUP. 
Table 7. Within-breed prediction accuracy when the heritability is doubled or tripled ${ }^{1}$

\begin{tabular}{|c|c|c|c|c|c|}
\hline Heritability & Method & Accuracy & Holstein & Jersey & AusRed $^{2}$ \\
\hline \multirow{4}{*}{0.6} & \multirow{2}{*}{ BayesR } & $2 \theta_{0.3}{ }^{3}$ & $0.82 \pm 0.02$ & $0.65 \pm 0.04$ & $0.43 \pm 0.08$ \\
\hline & & $2 \theta_{03 G}+\theta_{0.6 . P}{ }^{4}$ & $0.81 \pm 0.02$ & $0.65 \pm 0.04$ & $0.40 \pm 0.07$ \\
\hline & \multirow{2}{*}{ Genomic BLUP } & $2 \theta_{0.3}$ & $0.77 \pm 0.01$ & $0.65 \pm 0.04$ & $0.42 \pm 0.09$ \\
\hline & & $2 \theta_{0.3 . G}+\theta_{0.6 . P}$ & $0.76 \pm 0.01$ & $0.65 \pm 0.03$ & $0.40 \pm 0.07$ \\
\hline \multirow[t]{4}{*}{0.9} & BayesR & Cross-validation & $0.88 \pm 0.01$ & $0.78 \pm 0.01$ & $0.44 \pm 0.02$ \\
\hline & \multirow[t]{3}{*}{ Genomic BLUP } & Cross-validation & $0.88 \pm 0.01$ & $0.78 \pm 0.01$ & $0.44 \pm 0.02$ \\
\hline & & $3 \theta_{0.3}$ & $0.93 \pm 0.01$ & $0.82 \pm 0.04$ & $0.53 \pm 0.12$ \\
\hline & & $3 \theta_{0.3, G}+\theta_{0.9, P}$ & $0.92 \pm 0.01$ & $0.79 \pm 0.05$ & $0.50 \pm 0.06$ \\
\hline
\end{tabular}

${ }^{1}$ Deterministic predictions are compared with cross-validation accuracies, scenario with a heritability of 4,000 QTL.

${ }^{2}$ Australian Red.

${ }^{3}$ Accuracy estimated as $r=\sqrt{\frac{\theta}{1+\theta-r^{2} h^{2}}}$, where $\theta=2 \theta_{0.3}$.

${ }^{4}$ Accuracy estimated as $r=\sqrt{\frac{\theta}{1+\theta-r^{2} h^{2}}}$, where $\theta=2 \theta_{0.3, G}+\theta_{0.6, P}$.

${ }^{5}$ Accuracy estimated as $r=\sqrt{\frac{\theta}{1+\theta-r^{2} h^{2}}}$, where $\theta=3 \theta_{0.3}$.

${ }^{6}$ Accuracy estimated as $r=\sqrt{\frac{\theta}{1+\theta-r^{2} h^{2}}}$, where $\theta=3 \theta_{0.3, G}+\theta_{0.9, P}$.

GBLUP and Bayes R. As reported in previous studies (Goddard and Hayes, 2009; Habier et al., 2010; Liu et al., 2011), increasing the size of the reference population resulted in an increased prediction accuracy. Whereas, for Holstein, large reference populations are shared between countries (Lund et al., 2011), the size of the reference population is a much more limiting factor in breeds with smaller population sizes. Our results demonstrated that sharing reference populations between breeds could aid breeds that have a limited population size (Hozé et al., 2014) and all breeds for traits that are rarely measured, particularly for Bayes $\mathrm{R}$.

Within a breed, LD causes correlation between SNP and causal polymorphisms over a long distance. Consequently, the effective number of independent chromosome segments whose effects must be estimated is low (approximately 5,000 in Holsteins from Table 3). How- ever, a prediction equation derived from 1 breed will only predict well in another breed if the LD between the SNP and the causal polymorphisms is the same in both breeds. A Holstein reference predicts breeding values in Red Holstein better than in other breeds, presumably because LD is better preserved between the more closely related breeds. The SNP on the Illumina BovineHD chip are sufficiently close to preserve LD between breeds (de Roos et al., 2008), but this implies that, using a multibreed reference population, effects for a much larger number of effective chromosome segments must be estimated, which reduces the accuracy of prediction. In our simulation study, when the reference and validation populations were of different breeds, Bayes $\mathrm{R}$ gave higher accuracy than GBLUP. This can be explained because Bayes $\mathrm{R}$ places more weight on SNP close to the causal polymorphisms, whereas GB-

Table 8. Influence of the number of chromosomes on prediction accuracy [scenario with a heritability of 0.6 and a Holstein $(\mathrm{H})$ reference population]

\begin{tabular}{|c|c|c|c|c|c|}
\hline Method & $\begin{array}{l}\text { Validation } \\
\text { population }\end{array}$ & \multicolumn{2}{|c|}{$2,000 \mathrm{QTL}$} & \multicolumn{2}{|c|}{ 4,000 QTL } \\
\hline \multirow[t]{3}{*}{ Bayes R } & Holstein & $0.86 \pm 0.01$ & $0.83 \pm 0.01$ & $0.82 \pm 0.00$ & $0.79 \pm 0.01$ \\
\hline & Jersey & $0.63 \pm 0.04$ & $0.64 \pm 0.03$ & $0.53 \pm 0.02$ & $0.51 \pm 0.01$ \\
\hline & Australian Red & $0.68 \pm 0.03$ & $0.65 \pm 0.05$ & $0.50 \pm 0.07$ & $0.60 \pm 0.04$ \\
\hline \multirow[t]{2}{*}{ Genomic BLUP } & Holstein & $0.78 \pm 0.01$ & $0.70 \pm 0.01$ & $0.77 \pm 0.01$ & $0.70 \pm 0.01$ \\
\hline & Australian Red & $0.30 \pm 0.05$ & $0.33 \pm 0.07$ & $0.37 \pm 0.05$ & $0.32 \pm 0.05$ \\
\hline
\end{tabular}


LUP uses SNP over a wide segment of a chromosome to predict the effect of causal polymorphisms in that segment. This is equivalent to saying that Bayes R estimates the effects of fewer segments. However, as the number of causal polymorphisms increases, the number of effects that Bayes $\mathrm{R}$ must estimate also increases. Consequently, when the number of QTL approaches or exceeds the number of effective segments estimated by GBLUP, then Bayes $\mathrm{R}$ has no advantage over GBLUP (Daetwyler et al., 2010). This occurs within breed when there are 4,000 or 10,000 QTL for a single breed but not between breeds, because $M_{e}$ may be larger than 10,000. Thus, Bayes R outperforms GBLUP for prediction between breeds or when the number of QTL is small (van den Berg et al., 2015); this is consistent with the advantage of Bayes R over GBLUP for across-breed prediction that has been observed in real data (Hayes et al., 2009a; Erbe et al., 2012; Kemper et al., 2015).

In our simulation, we assumed that causative mutations had the same effect in all breeds, and all causative mutations were included in the data set. In real data, nonadditive QTL effects combined with differences in allele frequencies across breeds could result in different QTL effects in different breeds. When constructing a multibreed GRM, a way to account for differences in effects across breeds because of differences in allele frequencies may be to standardize genotypes based on breed-specific allele frequencies, as shown by Wientjes et al. (2015b). In our study, the simulated effects were the same in all breeds; therefore, we used the overall frequency of the breeds to construct a GRM rather than breed-specific allele frequencies. Even when full sequence data are used, not all causative mutations will be in the data set due to genotyping and imputation errors, as well as not considering factors such as structural variants. Furthermore, not all QTL are shared between breeds; as a result of these factors, the advantage of multibreed and across-breed prediction observed in this simulation study was larger than that observed in real data (Lund et al., 2014).

It would be desirable to know the increase of accuracy that can be expected from combining populations before performing the full analysis and sharing complete data sets. Therefore, we tested if $\theta$ obtained using 1 reference population could be used to predict the accuracy of the joint reference population. Within breed, this resulted in an overestimation of the accuracy. An explanation for this could be that doubling the reference population does not double the information coming from pedigree relationships. However, even when
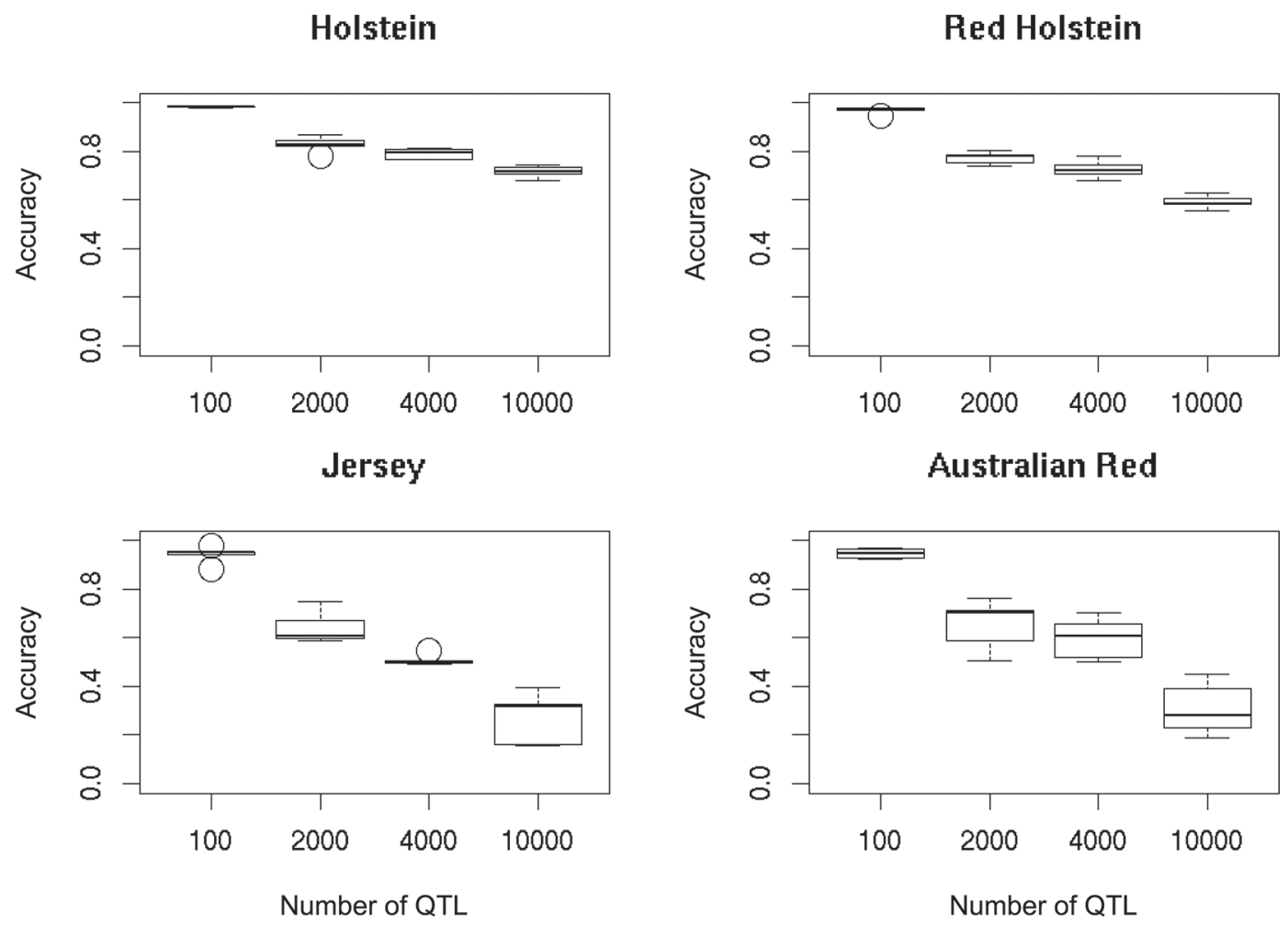

Figure 6. Influence of the number of QTL on prediction accuracy of Bayes R for scenarios with a heritability of $0.6,30$ chromosomes, and a reference population containing 7,989 Holstein individuals. The boxes show the middle $50 \%$ of values, the line shows the median, the whiskers show values outside the middle $50 \%$, and the circles are outliers. 
Holstein
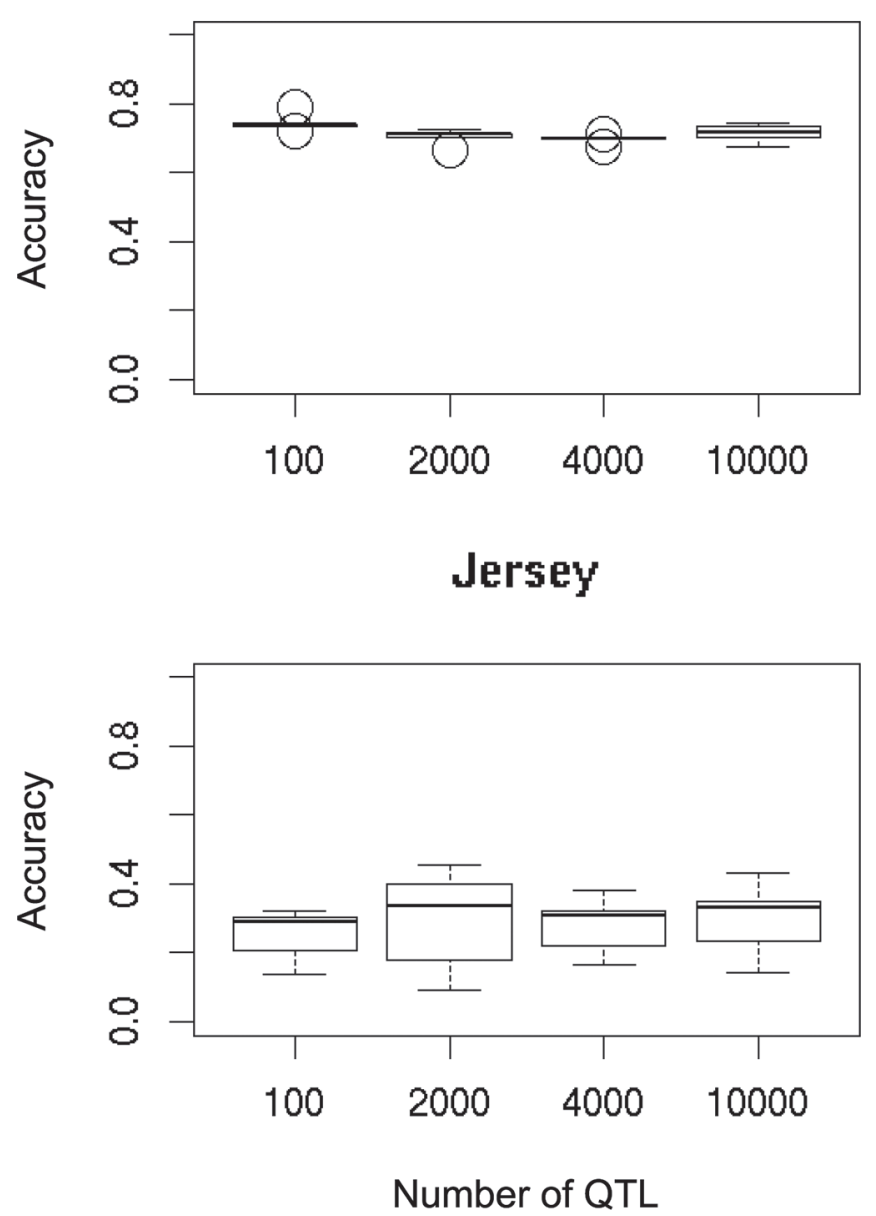

Red Holstein

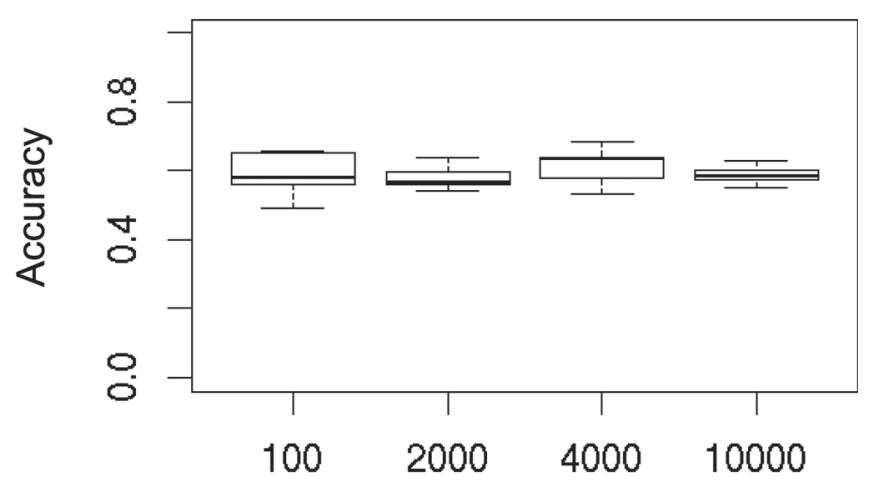

Australian Red

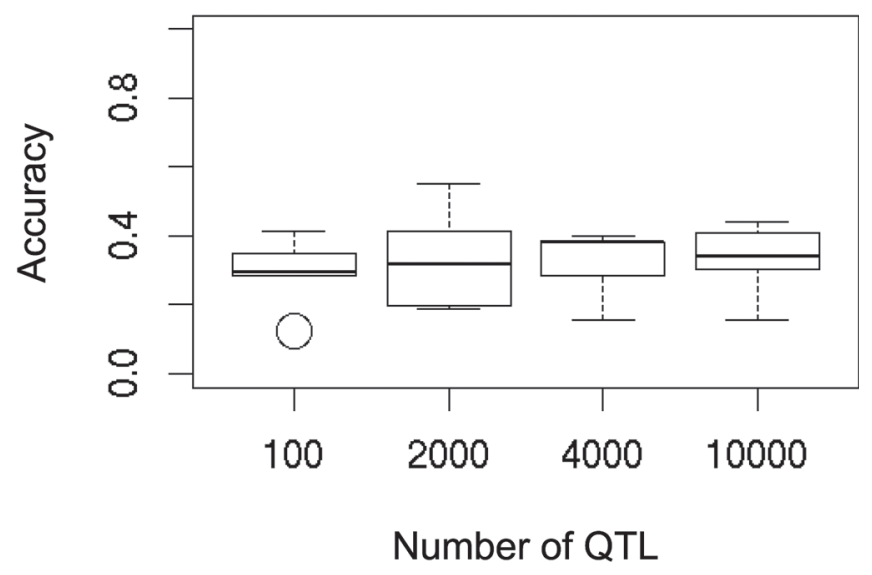

Figure 7. Influence of the number of QTL on prediction accuracy of genomic BLUP for scenarios with a heritability of $0.6,30$ chromosomes, and a reference population containing 7,989 Holstein individuals. The boxes show the middle 50\% of values, the line shows the median, the whiskers show values outside the middle $50 \%$, and the circles are outliers.

the pedigree information was removed, the contribution of genomic information was not proportional to the size of the reference population, perhaps because some relationships are not represented in the recorded pedigree. When reference populations from multiple breeds were combined, the deterministic accuracy of prediction based on adding up the $\theta$ obtained within breed was close to the accuracy obtained by cross-validation. This further indicates that the overestimation within breed was related to pedigree relationships that were negligible across breeds.

\section{Heritability}

What we have called the heritability is equivalent to the reliability of the phenotype to predict breeding value. Therefore, higher heritability can be used to model the benefits of multiple records on cows, a progeny test on bulls, or combining bull and cow data. Although the heritability increased the prediction accuracy regardless of the method, the increase was larger for Bayes $\mathrm{R}$ than for GBLUP, especially for across-breed prediction. The potential advantage of Bayesian variable selection models over GBLUP lies in being able to distinguish between QTL and variants that do not influence the trait, and the ability to do so improves when the heritability increases (van den Berg et al., 2013). This could explain why the advantage of Bayes $\mathrm{R}$ over GBLUP increased when the heritability increased. The use of $\theta$ to predict the effect of increased heritability worked well up to $\mathrm{h}^{2}=0.6$ but overestimated accuracy when $\mathrm{h}^{2}=0.9$. It appears that a very high heritability (i.e., more reliable phenotypes) is not as useful as adding more independent animals to the reference population, as more independent animals improves the identification of the segments whose effects need to be estimated. 


\section{The Size of the Genome and the Number of QTL}

Whereas decreasing the number of QTL had no effect on the accuracy of GBLUP, decreasing the number of chromosomes increased the accuracy of GBLUP but had no consistent effect on the accuracy of Bayes R. Reducing the number of chromosomes reduces $M_{e}$, thereby increasing the accuracy of GBLUP; however, the estimate of $M_{e}$ decreased only slightly (Table 3), probably because there are relationships among the animals that can be captured by half of the genome as well as by the whole genome. For Bayes $\mathrm{R}$, the $M_{e}$ is influenced both by the genome size and by the number of QTL. In our simulations, reducing the genome size did not reduce the number of QTL (which was large) and thus had little effect on the Bayes $\mathrm{R} M_{e}$. In the scenarios with a smaller number of QTL, the individual QTL effects were larger, whereas in scenarios with a large number of QTL, the individual QTL effects were small, making them more difficult to estimate by Bayes R. Consequently, the accuracy of Bayes R decreased when the number of QTL increased. This is line with results by van den Berg et al. (2013), where the ability of Bayes C to detect QTL decreased when the number of QTL increased.

\section{CONCLUSIONS}

Our results showed that, in real data sets, the GRM is likely to underestimate $M_{e}$ due to pedigree relationships among animals. Consequently, $M_{e}$ estimated from the GRM could not be used to predict the accuracy of genomic prediction in our data set. When $\theta$ is estimated for 1 data set using the accuracies obtained by crossvalidation and $r=\sqrt{\frac{\theta}{1+\theta-r^{2} h^{2}}}$, it can be used to approximately predict the accuracy obtained by increasing the number of individuals in the reference population or by changing the accuracy of their phenotypes. Separating out the pedigree contribution from the genomic contribution slightly improved the prediction of accuracy. The accuracy of multibreed prediction can be predicted by adding $\theta$ estimated from accuracies obtained by cross-validation using the single-breed reference populations for within- and across-breed prediction. Although the GRM could not be used to estimate $M_{e}$ in our data, the concept of effective number of chromosome segments is useful in understanding the interactions between multiple breeds, number of QTL, and method of analysis. Accuracy of GBLUP is unaffected by the number of QTL because it estimates effects for all chromosome segments weighted equally. When predicting from one breed to another, the number of effective chromosome segments increases greatly, resulting in low accuracy compared with within-breed prediction. Bayes R can be considered as a GBLUP in which SNP close to QTL are given greater weight. Consequently, if the number of QTL is small, the number of segments whose effects need to be estimated is small and the accuracy is increased. The $M_{e}$ with Bayes $\mathrm{R}$ tends to be in between the number of QTL and the number of effective segments when using GBLUP. However, if the number of QTL approaches the number of effective segments for GBLUP, then Bayes $\mathrm{R}$ has no advantage over GBLUP. This may be the case within breeds. The low accuracy of across-breed prediction observed in real data is also consistent with $M_{e}$ being very large.

\section{ACKNOWLEDGMENTS}

This research was supported by the Center for Genomic Selection in Animals and Plants (GenSAP), Tjele, Denmark, funded by The Danish Council for Strategic Research, Copenhagen, Denmark. We acknowledge DataGene, Bundoora, Victoria, Australia, and CRV Netherlands, Arnhem, the Netherlands, for providing access to data used in this study. We acknowledge our partners in the 1000 Bull Genomes Project, Bundoora, Victoria, Australia, for access to the reference genomes. We acknowledge Paul Stothard and the team at the University of Alberta, Edmonton, Canada, for collating annotation information of sequence variants used in this study.

\section{REFERENCES}

Brard, S., and A. Ricard. 2015. Is the use of formulae a reliable way to predict the accuracy of genomic selection? J. Anim. Breed. Genet. 132:207-217. https://doi.org/10.1111/jbg.12123.

Clark, S. A., J. M. Hickey, and J. H. J. van der Werf. 2011. Different models of genetic variation and their effect on genomic evaluation. Genet. Sel. Evol. 43:18. https://doi.org/10.1186/1297-9686-43-18.

Daetwyler, H. D., R. Pong-Wong, B. Villanueva, and J. A. Woolliams. 2010. The impact of genetic architecture on genome-wide evaluation methods. Genetics 185:1021-1031. https://doi.org/10.1534/ genetics.110.116855.

Daetwyler, H. D., B. Villanueva, and J. A. Woolliams. 2008. Accuracy of predicting the genetic risk of disease using a genome-wide approach. PLoS One 3:e3395. https://doi.org/10.1371/journal.pone .0003395 .

de Roos, A. P. W., B. J. Hayes, and M. E. Goddard. 2009. Reliability of genomic predictions across multiple populations. Genetics 183:1545-1553. https://doi.org/10.1534/genetics.109.104935.

de Roos, A. P. W., B. J. Hayes, R. J. Spelman, and M. E. Goddard. 2008. Linkage disequilibrium and persistence of phase in HolsteinFriesian, Jersey and Angus cattle. Genetics 179:1503-1512. https: //doi.org/10.1534/genetics.107.084301.

Druet, T., I. M. Macleod, and B. J. Hayes. 2014. Toward genomic prediction from whole-genome sequence data: Impact of sequencing design on genotype imputation and accuracy of predictions. Heredity 112:39-47. https://doi.org/10.1038/hdy.2013.13.

Erbe, M., B. Gredler, F. R. Seefried, B. Bapst, and H. Simianer. 2013. A function accounting for training set size and marker density 
to model the average accuracy of genomic prediction. PLoS One 8:e81046.

Erbe, M., B. J. Hayes, L. K. Matukumalli, S. Goswami, P. J. Bowman, C. M. Reich, B. A. Mason, and M. E. Goddard. 2012. Improving accuracy of genomic predictions within and between dairy cattle breeds with imputed high-density single nucleotide polymorphism panels. J. Dairy Sci. 95:4114-4129. https://doi.org/10.3168/jds $.2011-5019$.

Gilmour, A. R., B. J. Gogel, B. R. Cullis, R. Thompson, D. Butler, M. Cherry, D. Collins, G. Dutkowski, S. A. Harding, and K. Haskard. 2008. ASReml User Guide Release 3.0. VSN International Ltd., Hemel Hempstead, United Kingdom.

Goddard, M. 2009. Genomic selection: Prediction of accuracy and maximisation of long term response. Genetica 136:245-257. https: //doi.org/10.1007/s10709-008-9308-0.

Goddard, M. E., and B. J. Hayes. 2009. Mapping genes for complex traits in domestic animals and their use in breeding programmes. Nat. Rev. Genet. 10:381-391. https://doi.org/10.1038/nrg2575.

Goddard, M. E., B. J. Hayes, and T. H. E. Meuwissen. 2011. Using the genomic relationship matrix to predict the accuracy of genomic selection. J. Anim. Breed. Genet. 128:409-421. https://doi.org/10 $.1111 / \mathrm{j} .1439-0388.2011 .00964 . x$.

Habier, D., R. L. Fernando, and J. C. M. Dekkers. 2007. The impact of genetic relationship information on genome-assisted breeding values. Genetics 177:2389-2397. https://doi.org/10.1534/genetics .107 .081190

Habier, D., J. Tetens, F.-R. Seefried, P. Lichtner, and G. Thaller. 2010. The impact of genetic relationship information on genomic breeding values in German Holstein cattle. Genet. Sel. Evol. 42:5. https: //doi.org/10.1186/1297-9686-42-5.

Hayes, B. J., P. J. Bowman, A. C. Chamberlain, K. Verbyla, and M. E. Goddard. 2009a. Accuracy of genomic breeding values in multibreed dairy cattle populations. Genet. Sel. Evol. 41:51. https://doi .org/10.1186/1297-9686-41-51.

Hayes, B. J., P. M. Visscher, and M. E. Goddard. 2009b. Increased accuracy of artificial selection by using the realized relationship matrix. Genet. Res. (Camb.) 91:47-60. https://doi.org/10.1017/ S0016672308009981.

Hozé, C., S. Fritz, F. Phocas, D. Boichard, V. Ducrocq, and P. Croiseau. 2014. Efficiency of multi-breed genomic selection for dairy cattle breeds with different sizes of reference population. J. Dairy Sci. 97:3918-3929. https://doi.org/10.3168/jds.2013-7761.

Kemper, K. E., C. M. Reich, P. J. Bowman, C. J. Vander Jagt, A. J. Chamberlain, B. A. Mason, B. J. Hayes, and M. E. Goddard. 2015. Improved precision of QTL mapping using a nonlinear Bayesian method in a multi-breed population leads to greater accuracy of across-breed genomic predictions. Genet. Sel. Evol. 47:29. https:// doi.org/10.1186/s12711-014-0074-4.

Lee, S. H., S. Clark, and J. H. J. Van Der Werf. 2017. Estimation of genomic prediction accuracy from reference populations with varying degrees of relationship. PLoS One 12:e0189775. https://doi.org/10 .1371/journal.pone.0189775.

Liu, Z., F. R. Seefried, F. Reinhardt, S. Rensing, G. Thaller, and R. Reents. 2011. Impacts of both reference population size and inclusion of a residual polygenic effect on the accuracy of genomic prediction. Genet. Sel. Evol. 43:19. https://doi.org/10.1186/1297 -9686-43-19.

Lund, M. S., A. P. W. de Roos, A. G. de Vries, T. Druet, V. Ducrocq, S. Fritz, F. Guillaume, B. Guldbrandtsen, Z. Liu, R. Reents, C. Schrooten, F. Seefried, and G. Su. 2011. A common reference population from four European Holstein populations increases reliability of genomic predictions. Genet. Sel. Evol. 43:43. https://doi .org/10.1186/1297-9686-43-43.

Lund, M. S., G. Su, L. Janss, B. Guldbrandtsen, and R. F. Brøndum. 2014. Invited review: Genomic evaluation of cattle in a multi-breed context. Livest. Sci. 166:101-110. https://doi.org/10.1016/j.livsci 2014.05.008

MacLeod, I. M., P. J. Bowman, C. J. Vander Jagt, M. Haile-Mariam, K. E. Kemper, A. J. Chamberlain, C. Schrooten, B. J. Hayes, and
M. E. Goddard. 2016. Exploiting biological priors and sequence variants enhances QTL discovery and genomic prediction of complex traits. BMC Genomics 17:144. https://doi.org/10.1186/ s12864-016-2443-6.

Meuwissen, T., and M. Goddard. 2010. Accurate prediction of genetic values for complex traits by whole-genome resequencing. Genetics 185:623-631. https://doi.org/10.1534/genetics.110.116590.

Meuwissen, T., B. Hayes, and M. Goddard. 2013. Accelerating improvement of livestock with genomic selection. Annu. Rev. Anim. Biosci. 1:221-237. https://doi.org/10.1146/annurev-animal-031412 $-103705$.

Meuwissen, T. H., B. J. Hayes, and M. E. Goddard. 2001. Prediction of total genetic value using genome-wide dense marker maps. Genetics 157:1819-1829.

Misztal, I. 2016. Inexpensive computation of the inverse of the genomic relationship matrix in populations with small effective population size. Genetics 202:401-409. https://doi.org/10.1534/genetics.115 .182089.

Pocrnic, I., D. A. L. Lourenco, Y. Masuda, A. Legarra, and I. Misztal. 2016a. The dimensionality of genomic information and its effect on genomic prediction. Genetics 203:573-581. https://doi.org/10 .1534 /genetics.116.187013.

Pocrnic, I., D. A. L. Lourenco, Y. Masuda, and I. Misztal. 2016b. Dimensionality of genomic information and performance of the algorithm for proven and young for different livestock species. Genet. Sel. Evol. 48:82. https://doi.org/10.1186/s12711-016-0261-6.

R Core Team. 2015. R: A language and environment for statistical computing. R Foundation for Statistical Computing, Vienna, Austria. http://www.R-project.org/.

van den Berg, I., S. Fritz, and D. Boichard. 2013. QTL fine mapping with Bayes $C(\pi)$ : A simulation study. Genet. Sel. Evol. 45:19.

van den Berg, S., M. P. L. Calus, T. H. E. Meuwissen, and Y. C. J. Wientjes. 2015. Across population genomic prediction scenarios in which Bayesian variable selection outperforms GBLUP. BMC Genet. 16:146. https://doi.org/10.1186/s12863-015-0305-x.

Vandenplas, J., J. J. Windig, and M. P. L. Calus. 2017. Prediction of the reliability of genomic breeding values for crossbred performance. Genet. Sel. Evol. 49:43. https://doi.org/10.1186/s12711 $-017-0318-1$.

Wang, T., Y. P. P. Chen, P. J. Bowman, M. E. Goddard, and B. J. Hayes. 2016. A hybrid expectation maximisation and MCMC sampling algorithm to implement Bayesian mixture model based genomic prediction and QTL mapping. BMC Genomics 17:744. https://doi.org/10.1186/s12864-016-3082-7.

Wientjes, Y. C., M. P. Calus, M. E. Goddard, and B. J. Hayes. 2015a. Impact of QTL properties on the accuracy of multi-breed genomic prediction. Genet. Sel. Evol. 47:42. https://doi.org/10.1186/ s12711-015-0124-6.

Wientjes, Y. C., R. F. Veerkamp, P. Bijma, H. Bovenhuis, C. Schrooten, and M. P. Calus. 2015b. Empirical and deterministic accuracies of across-population genomic prediction. Genet. Sel. Evol. 47:5 https://doi.org/10.1186/s12711-014-0086-0.

Wientjes, Y.C.J., P. Bijma, R.F. Veerkamp, and M.P.L. Calus. 2015c. An equation to predict the accuracy of genomic values by combining data from multiple traits, populations, or environments. Genetics 202:799-823.

Wientjes, Y. C. J., R. F. Veerkamp, and M. P. L. Calus. 2013. The effect of linkage disequilibrium and family relationships on the reliability of genomic prediction. Genetics 193:621-631. https:// doi.org/10.1534/genetics.112.146290.

Yang, J., S. H. Lee, M. E. Goddard, and P. M. Visscher. 2011. GCTA: A tool for genome-wide complex trait analysis. Am. J. Hum. Genet. 88:76-82. https://doi.org/10.1016/j.ajhg.2010.11.011.

Young, C. W., and A. J. Seykora. 1996. Estimates of inbreeding and relationship among registered Holstein females in the United States. J. Dairy Sci. 79:502-505. 


\section{APPENDIX 1}

Table A1. Number of simulated QTL segregating in each breed ${ }^{1}$

\begin{tabular}{lrrr}
\hline QTL size & $\mathrm{HH}^{2}$ & \multicolumn{1}{c}{$\mathrm{JJ}^{3}$} & $\mathrm{RR}^{4}$ \\
\hline Large & 15 & 15 & 14 \\
Medium & 500 & 478 & 454 \\
Small & 3,482 & 3,353 & 3,178 \\
\hline
\end{tabular}

${ }^{1}$ For the scenario with 4,000 QTL, numbers in the table are averaged over 5 repeats.

${ }^{2}$ Reference population containing 15,547 Holstein individuals.

${ }^{3}$ Reference population containing 4,310 Jersey individuals.

${ }^{4}$ Reference population containing 769 Australian Red individuals.

$H H$, large QTL

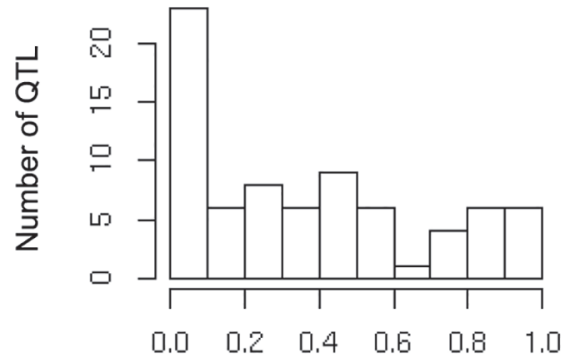

HH, medium QTL

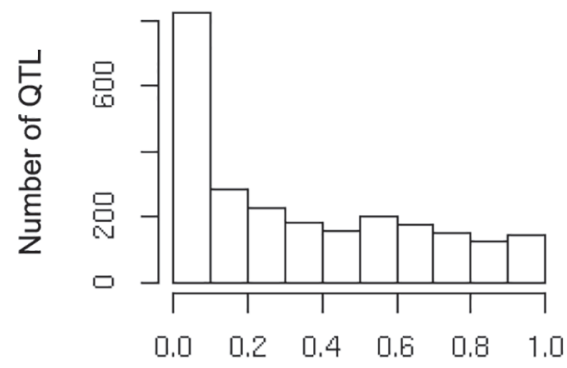

HH, small QTL

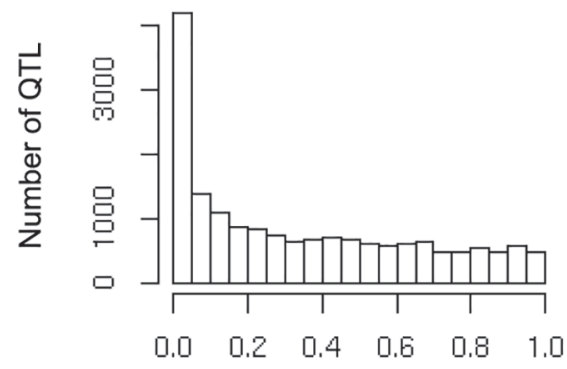

Allele frequency
JJ. large QTL

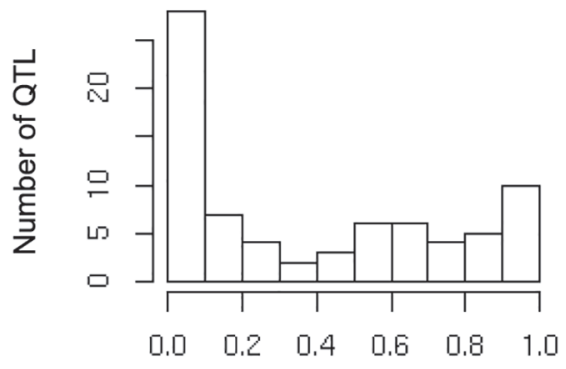

Jل, medium QTL

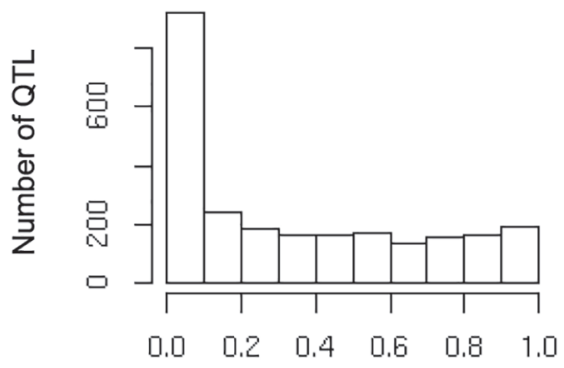

JJ. small QTL

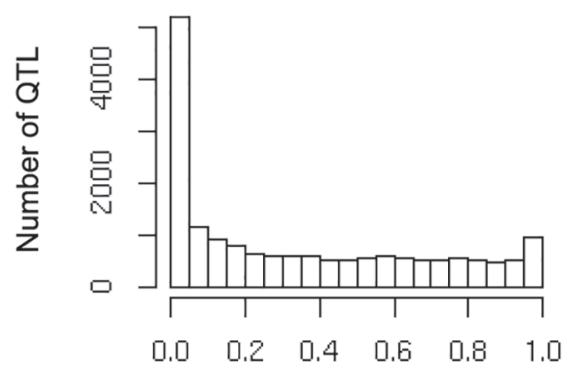

Allele frequency
RR, large QTL

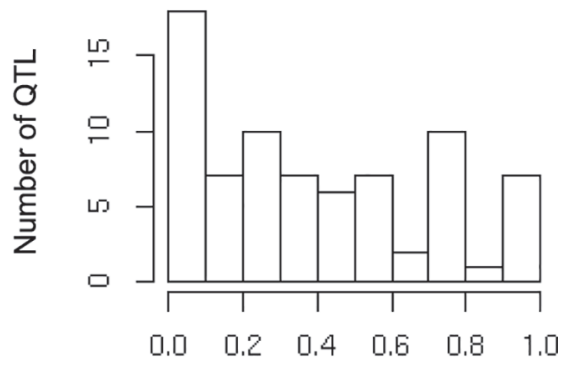

RP, medium QTL

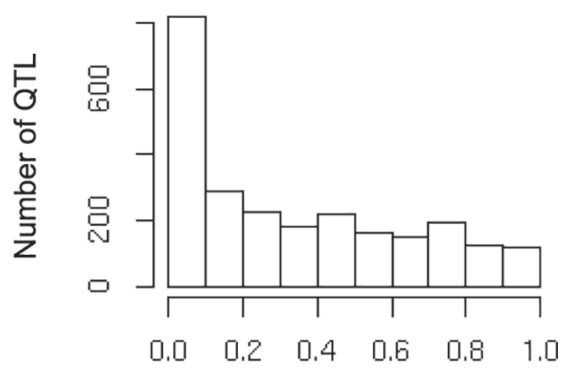

RR. small QTL

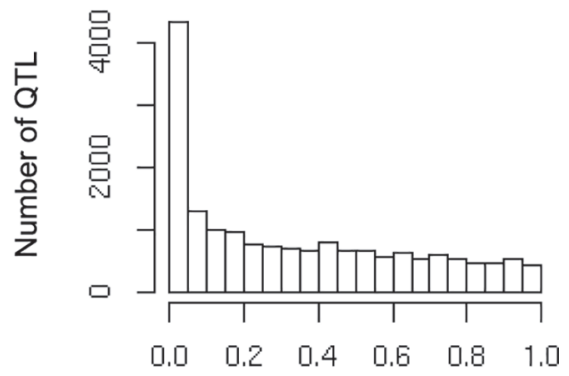

Allele frequency

Figure A1. Allele frequencies of simulated QTL per breed. HH = reference population containing 15,547 Holstein individuals; JJ = reference population containing 4,310 Jersey individuals; $\mathrm{RR}=$ reference population containing 769 Australian Red individuals. 
APPENDIX 2

Table A2. Estimated heritabilities (using genomic BLUP) within each reference population

\begin{tabular}{lcccccccc}
\hline $\mathrm{h} 2 \_\operatorname{sim}^{1}$ & $\mathrm{H}^{2}$ & $\mathrm{HH}^{3}$ & $\mathrm{~J}^{4}$ & $\mathrm{JJ}^{5}$ & $\mathrm{R}^{6}$ & $\mathrm{RR}^{7}$ & $\mathrm{H}^{+} \mathrm{J}^{8}$ & $\mathrm{H}^{+} \mathrm{J}+\mathrm{RR}^{9}$ \\
\hline 0.3 & 0.31 & 0.30 & 0.29 & 0.32 & 0.22 & 0.28 & 0.30 & 0.30 \\
0.6 & 0.64 & 0.63 & 0.69 & 0.67 & 0.62 & 0.70 & 0.64 & 0.63 \\
0.9 & 0.93 & 0.92 & 0.95 & 0.95 & 0.84 & 0.90 & 0.93 & 0.93 \\
\hline
\end{tabular}

${ }^{1}$ Simulated heritability.

${ }^{2}$ Reference population containing 7,989 Holstein individuals.

${ }^{3}$ Reference population containing 15,547 Holstein individuals.

${ }^{4}$ Reference population containing 2,323 Jersey individuals.

${ }^{5}$ Reference population containing 4,310 Jersey individuals.

${ }^{6}$ Reference population containing 384 Australian Red individuals.

${ }^{7}$ Reference population containing 769 Australian Red individuals.

${ }^{8}$ Reference population containing 7,989 Holstein and 2,323 Jersey individuals.

${ }^{9}$ Reference population containing 7,989 Holstein, 2,323 Jersey, and 769 Australian Red individuals.

Table A3. Ranking of breeding values using a reference population containing 7,989 Holstein (H), 15,547 Holstein (HH), 2,323 Jersey (J), 4,310 Jersey (JJ), 348 Australian Red (R), 769 Australian Red (RR), 7,989 Holstein and 2,323 Jersey $(\mathrm{H}+\mathrm{J})$, or 7,989 Holstein, 2,323 Jersey, and 769 Australian Red $(\mathrm{H}+\mathrm{J}+\mathrm{RR})$ individuals, for the 10 individuals of the Holstein validation population that had the largest breeding values using the $\mathrm{H}+\mathrm{J}$ reference population in the first repeat ${ }^{1}$

\begin{tabular}{|c|c|c|c|c|c|c|c|}
\hline $\mathrm{H}+\mathrm{J}$ & $\mathrm{H}$ & $\mathrm{HH}$ & $\mathrm{J}$ & JJ & $\mathrm{R}$ & $\mathrm{RR}$ & $\mathrm{H}+\mathrm{J}+\mathrm{RR}$ \\
\hline 1 & 2 & 2 & 6 & 1 & 121 & 257 & 1 \\
\hline 2 & 1 & 1 & 192 & 33 & 365 & 360 & 2 \\
\hline 3 & 7 & 22 & 4 & 2 & 216 & 256 & 4 \\
\hline 4 & 6 & 4 & 209 & 206 & 340 & 148 & 7 \\
\hline 5 & 5 & 9 & 120 & 77 & 513 & 192 & 3 \\
\hline 6 & 3 & 3 & 262 & 75 & 27 & 4 & 5 \\
\hline 7 & 4 & 16 & 26 & 145 & 371 & 49 & 12 \\
\hline 8 & 11 & 7 & 245 & 254 & 348 & 177 & 8 \\
\hline 9 & 9 & 10 & 58 & 316 & 231 & 210 & 11 \\
\hline 10 & 8 & 37 & 8 & 11 & 204 & 229 & 16 \\
\hline
\end{tabular}

${ }^{1}$ Results for the other 4 repeats and genomic BLUP showed similar trends.

Table A4. Ranking of breeding values using a reference population containing 7,989 Holstein (H), 15,547 Holstein (HH), 2,323 Jersey (J), 4,310 Jersey (JJ), 348 Australian Red (R), 769 Australian Red (RR), 7,989 Holstein and 2,323 Jersey $(\mathrm{H}+\mathrm{J})$, or 7,989 Holstein, 2,323 Jersey, and 769 Australian Red $(\mathrm{H}+\mathrm{J}+\mathrm{RR})$ individuals, for the 10 individuals of the Jersey validation population that had the largest breeding values using the $\mathrm{H}+\mathrm{J}$ reference population in the first repeat ${ }^{1}$

\begin{tabular}{lrrrrrrr}
\hline $\mathrm{H}+\mathrm{J}$ & $\mathrm{H}$ & $\mathrm{HH}$ & $\mathrm{J}$ & $\mathrm{JJ}$ & $\mathrm{R}$ & $\mathrm{RR}$ & $\mathrm{H}+\mathrm{J}+\mathrm{RR}$ \\
\hline 1 & 23 & 64 & 17 & 37 & 207 & 299 & 4 \\
2 & 26 & 17 & 9 & 23 & 460 & 414 & 6 \\
3 & 28 & 4 & 2 & 8 & 490 & 198 & 5 \\
4 & 137 & 42 & 5 & 3 & 527 & 548 & 2 \\
5 & 1 & 1 & 4 & 7 & 11 & 462 & 1 \\
6 & 13 & 8 & 11 & 6 & 184 & 348 & 7 \\
7 & 22 & 3 & 92 & 124 & 373 & 362 & 3 \\
8 & 174 & 161 & 18 & 19 & 216 & 343 & 9 \\
9 & 61 & 19 & 7 & 1 & 218 & 457 & 11 \\
10 & 122 & 114 & 19 & 28 & 411 & 517 & \\
\hline
\end{tabular}

${ }^{1}$ Results for the other 4 repeats and genomic BLUP showed similar trends. 
Table A5. Ranking of breeding values using a reference population containing 7,989 Holstein (H), 15,547 Holstein (HH), 2,323 Jersey (J), 4,310 Jersey (JJ), 348 Australian Red (R), 769 Australian Red (RR), 7,989 Holstein and 2,323 Jersey $(\mathrm{H}+\mathrm{J})$, or 7,989 Holstein, 2,323 Jersey, and 769 Australian Red $(\mathrm{H}+\mathrm{J}+\mathrm{RR})$ individuals, for the 10 individuals of the Red Holstein validation population that had the largest breeding values using the $\mathrm{H}+\mathrm{J}$ reference population in the first repeat ${ }^{1}$

\begin{tabular}{lrrrrrrr}
\hline $\mathrm{H}+\mathrm{J}$ & $\mathrm{H}$ & $\mathrm{HH}$ & $\mathrm{J}$ & $\mathrm{JJ}$ & $\mathrm{R}$ & $\mathrm{RR}$ & $\mathrm{H}+\mathrm{J}+\mathrm{RR}$ \\
\hline 1 & 1 & 1 & 247 & 182 & 358 & 614 & 1 \\
2 & 6 & 7 & 26 & 62 & 434 & 26 & 2 \\
3 & 2 & 9 & 114 & 59 & 331 & 95 & 4 \\
4 & 14 & 5 & 98 & 47 & 665 & 391 & 5 \\
5 & 5 & 3 & 454 & 480 & 289 & 99 & 6 \\
6 & 8 & 2 & 148 & 217 & 616 & 507 & 25 \\
7 & 3 & 55 & 451 & 196 & 217 & 777 & 8 \\
8 & 12 & 18 & 460 & 493 & 722 & 718 & 5 \\
9 & 11 & 4 & 235 & 33 & 149 & 56 & 601 \\
10 & 9 & 34 & 305 & 88 & 130 & & 9 \\
\hline
\end{tabular}

${ }^{1}$ Results for the other 4 repeats and genomic BLUP showed similar trends.

Table A6. Ranking of breeding values using a reference population containing 7,989 Holstein (H), 15,547 Holstein (HH), 2,323 Jersey (J), 4,310 Jersey (JJ), 348 Australian Red (R), 769 Australian Red (RR), 7,989 Holstein and 2,323 Jersey $(\mathrm{H}+\mathrm{J})$, or 7,989 Holstein, 2,323 Jersey, and 769 Australian Red $(\mathrm{H}+\mathrm{J}+\mathrm{RR})$ individuals, for the 10 individuals of the Australian Red validation population that had the largest breeding values using the $\mathrm{H}+\mathrm{J}$ reference population in the first repeat ${ }^{1}$

\begin{tabular}{lrrrrrrr}
\hline $\mathrm{H}+\mathrm{J}$ & $\mathrm{H}$ & $\mathrm{HH}$ & $\mathrm{J}$ & $\mathrm{JJ}$ & $\mathrm{R}$ & $\mathrm{RR}$ & $\mathrm{H}+\mathrm{J}+\mathrm{RR}$ \\
\hline 1 & 1 & 5 & 4 & 7 & 9 & 4 & 1 \\
2 & 10 & 6 & 22 & 24 & 13 & 5 & 2 \\
3 & 2 & 1 & 46 & 2 & 27 & 34 & 3 \\
4 & 3 & 3 & 61 & 30 & 10 & 8 & 5 \\
5 & 5 & 2 & 7 & 11 & 41 & 26 & 8 \\
6 & 13 & 7 & 36 & 60 & 20 & 22 & 4 \\
7 & 4 & 9 & 78 & 52 & 6 & 11 & 1 \\
8 & 7 & 12 & 27 & 9 & 1 & 47 & 6 \\
9 & 11 & 4 & 35 & 15 & 31 & 2 & 4 \\
10 & 6 & 31 & 51 & 45 & 3 & &
\end{tabular}

${ }^{1}$ Results for the other 4 repeats and genomic BLUP showed similar trends. 